\title{
The updated spectral catalogue of INTEGRAL gamma-ray bursts
}

\author{
G. Vianello ${ }^{1,2}$, D. Götz ${ }^{3}$, and S. Mereghetti ${ }^{1}$ \\ 1 INAF - Istituto di Astrofisica Spaziale e Fisica Cosmica Milano, via Bassini 15, 20133 Milano, Italy \\ e-mail: vianello@iasf-milano.inaf.it \\ 2 Dipartimento di Fisica e Matematica, Università dell'Insubria, via Valleggio 11, 22100 Como, Italy \\ 3 CEA-Saclay, DSM/Irfu/Service d'Astrophysique, Orme des Merisiers, 91191 Gif-sur-Yvette, France
}

Received 29 October 2008 / Accepted 12 December 2008

\section{ABSTRACT}

\begin{abstract}
We present a catalogue with the properties of all the bursts detected and localized by the IBIS instrument onboard the INTEGRAL satellite from November 2002 to September 2008. The sample is composed of 56 bursts, corresponding to a rate of $\sim 0.8$ GRB per month. Thanks to the performances of the INTEGRAL Burst Alert System, 50\% of the IBIS GRBs have detected afterglows, while 5\% have redshift measurements. A spectral analysis of the 43 bursts in the INTEGRAL public archive has been carried out using the most recent software and calibration, deriving an updated, homogeneous and accurate catalogue with the spectral features of the sample. When possible a time-resolved spectral analysis also has been carried out. The GRBs in the sample have 20-200 keV fluences in the range $5 \times 10^{-8}-2.5 \times 10^{-4} \mathrm{erg} \mathrm{cm}^{-2}$, and peak fluxes in the range $0.11-56 \mathrm{ph} \mathrm{cm}^{-2} \mathrm{~s}^{-1}$. While most of the spectra are well fitted by a power law with photon index $\sim 1.6$, we found that 9 bursts are better described by a cut-off power law, resulting in $E_{\mathrm{p}}$ values in the range 35-190 keV. Altough these results are comparable to those obtained with BAT onboard Swift, there is marginal evidence that ISGRI detects dimmer bursts than Swift/BAT. Using the revised spectral parameters and an updated sky exposure map that also takes into account the effects of the GRB trigger efficiency, we strengthen the evidence for a spatial correlation with the super galactic plane of the faint bursts with long spectral lag.
\end{abstract}

Key words. gamma rays: bursts - methods: data analysis - catalogs

\section{Introduction}

Enormous progress in the study of Gamma-Ray Bursts (GRBs) has been achieved after the results of the Satellite per Astronomia X (the Italian for "Satellite for X-ray Astronomy") BeppoSAX, that enabled the discovery of their counterparts at lower energy (Costa et al. 1997; van Paradijs et al. 1997; Frail et al. 1997). Our understanding of GRBs has mainly profited from dedicated instruments, or even satellites, specifically designed for the study of these enigmatic sources. For example, thanks to the very large GRB sample obtained with the Burst And Transient Sources Experiment (BATSE) onboard the Compton Gamma-Ray Observatory (CGRO), we learned that GRBs are isotropically distributed across the sky (Briggs et al. 1996), and that their $\log N-\log S$ is compatible with a cosmological origin (Meegan et al. 1992). BATSE also provided accurate spectra (e.g. Band et al. 1993), and confirmed that GRBs can be divided in two categories as a function of their duration and hardness, with about $25 \%$ of the GRBs being shorter than $2 \mathrm{~s}$ and harder compared to bursts of longer duration (Kouveliotou et al. 1993). Quick localizations with the High Energy Transient Explorer (HETE-2) satellite allowed investigators to study the GRB emission in X-rays (e.g. Vanderspek et al. 2004), and to detect the first afterglow of a short GRB (Fox \& React GRB Team 2005). Swift (Gehrels et al. 2004), launched in 2004, is currently localizing about 100 bursts per year, and is able to investigate the early phases of the X-ray afterglow, which were unaccessible to former instrumentation. Many key parameters are derived from the afterglow observations. Among them one of the most important is the distance, which is essential to infer the intrinsic energy and luminosity. GRBs are the most powerful explosions in the Universe, with isotropic equivalent energy $E_{\text {iso }}$ in the range from $10^{50}$ to $10^{54} \mathrm{erg}$ (Amati 2006), although such values can be reduced with the hypothesis that GRBs are collimated sources (Rhoads 1997), as supported by the detection of achromatic breaks in some afterglow light curves. The jet opening angles inferred from the afterglow break times (Sari et al. 1999) indicate lower energy values, clustered around $10^{51}$ erg with a reduced spread (Frail et al. 2001; Bloom et al. 2003). However, the observation of non-achromatic breaks in some afterglows, and the apparent lack of any kind of break in some others, casts doubts on this argument (see Curran et al. 2007, and reference therein). In only a few years, the Swift satellite has more than doubled the number of GRBs with known redshift, and discovered the most distant burst to date at $z \sim 6.7$, (GRB 080913, Fynbo et al. 2008).

Recent progresses in GRB science concern also the identification of their progenitors. There is general consensus in considering the collapse of massive stars $\left(>30 M_{\odot}\right)$ as the origin of long GRBs (Woosley 1993; Vietri \& Stella 1998). Indeed, in many cases late-time bumps in the afterglow light curves of long GRBs have been reported (e.g. Bloom et al. 1999; Zeh et al. 2004), and are interpreted as an emerging supernova component. In a handful of cases the GRB/SN association has been spectroscopically confirmed (e.g. Malesani et al. 2004a). However, in some cases deep optical follow-up observations failed to detect SN signatures from nearby long GRBs (e.g. Della Valle et al. 2006), indicating the possible existence of a different class of long GRBs. Models involving the merging of two compact objects (black holes, neutron stars, or white dwarfs) are, on the other hand, preferred for short GRBs (Perna \& Belczynski 2002).

Besides GRB-dedicated missions, also "general purpose" $\mathrm{X}$-ray/gamma-ray satellites can significantly contribute to the 
GRB quest. The most remarkable example is indeed the aforementioned BeppoSAX mission, that thanks to its rapid and accurate localizations led to the discovery of afterglows at X-ray, optical, and radio wavelengths, firmly proving the cosmological origin of these sources by allowing a measurement of their redshift.

The International Gamma-Ray Astrophysics Laboratory (INTEGRAL, Winkler et al. 2003), launched in October 2002, carries a set of coded mask instruments dedicated to fine imaging and spectroscopy in the soft $\gamma$-ray energy domain $(15 \mathrm{keV}-10 \mathrm{MeV})$. Even if INTEGRAL is not a mission specifically dedicated to GRBs, its imager instrument IBIS (Imager on Board the Integral Satellite, Ubertini et al. 2003a), thanks to a large field of view $\left(29^{\circ} \times 29^{\circ}\right)$ and a good point source location accuracy $\left(\sim 1-2^{\prime}\right)$, is well suited for GRB studies, in particular with its low energy detector plane ISGRI (INTEGRAL Soft Gamma-Ray Imager, Lebrun et al. 2003) (15 keV-1 MeV) . Pre-launch expectations of detecting about one GRB per month in the IBIS field of view prompted the development of the INTEGRAL Burst Alert System (IBAS).

IBAS (Mereghetti et al. 2003b) is a software system, running on the ground at the INTEGRAL Science Data Centre (ISDC; Courvoisier et al. 2003), able to detect and localize in real time the GRBs in the IBIS field of view. Thanks to IBAS, the INTEGRAL satellite has been the first to provide GRB positions with an uncertainty of only $\sim 2-3$ arcmin in near real time (less than a few tens of second after the burst trigger). To date 56 bursts have been detected in IBIS/ISGRI data ${ }^{1}$, mostly automatically by IBAS, and a few in off-line searches.

In this paper we present an updated catalog of all the GRBs localized with INTEGRAL, with a detailed spectral analysis of the ones for which public data are available. Some results have been published already by different authors on individual bursts, especially the ones discovered in the first months of the mission. More recently, a comprehensive analysis of a sample of 46 INTEGRAL bursts has been presented by Foley et al. (2008), who focused mainly on their timing properties. They also extract spectra of some GRBs, but using an old version of the software and of the calibration that now we know to be not adequate when dealing with off-axis bursts. The main purpose of our work is to provide a homogeneous set of results, obtained with the latest instrument calibration and software, focusing mainly on the spectral properties. In several cases the spectral parameters we derived differ from, and supersede, those reported in previous analyses, that were based on earlier software versions and calibrations.

\section{Data analysis}

\subsection{The IBIS coded-mask telescope}

IBIS (Ubertini et al. 2003b) is an imaging gamma-ray telescope, based on the coded-mask technique, with a square field of view of $29^{\circ} \times 29^{\circ}$ (at zero sensitivity). The sensitivity is maximum and nearly uniform within the inner $8.5^{\circ} \times 8.5^{\circ}$, corresponding to the so called fully coded field of view (FCFOV). Our analysis is mainly based on data taken with the IBIS low energy detector ISGRI (Lebrun et al. 2003), consisting of an array of $128 \times 128 \mathrm{CdTe}$ crystals sensitive in the nominal $15 \mathrm{keV}-1 \mathrm{MeV}$ energy range. The CdTe detector thickness of $2 \mathrm{~mm}$ ensures an efficiency of $50 \%$ at $150 \mathrm{keV}$. Above this energy the efficiency decreases rapidly (10\% at $300 \mathrm{keV})$, and although the brightest

${ }^{1}$ See http://ibas.iasf-milano.inaf.it
GRBs are detected up to $\sim 500 \mathrm{keV}$, the majority of the spectra presented here refer only to the $18-300 \mathrm{keV}$ band. The central part of the IBIS field of view is covered also by the Joint European X-ray Monitor (JEM-X, Lund et al. 2003) and the Optical Monitoring Camera (OMC, Mas-Hesse et al. 2003). When possible we have also analyzed data from these instruments.

\subsection{GRB sample}

In Table 2 we list the 56 GRBs observed with ISGRI until now (end of September 2008). Most of them were discovered and localized with the INTEGRAL Burst Alert System (IBAS; Mereghetti et al. 2003b) and their coordinates were automatically distributed in near real time within a few seconds. The remaining ones were detected during the initial IBAS performance verification phase (when automated alert distribution was not yet enabled), in off-line searches, or after interactive verification of low significance IBAS internal alerts.

The sky distribution of the 56 bursts is plotted in Fig. 1. The concentration of events close to the galactic plane simply reflects the greater exposure devoted to these regions by the pointing program of INTEGRAL. The IBAS trigger efficiency is maximum in the fully coded field of view, where all of the detector is exposed to the flux of the source and the signal to noise ratio is maximized. Towards larger off-axis angle the efficiency decreases, so that we need a brighter burst to obtain the same signal-to-noise. On the other hand the sky area covered by the detector increases. Thus it is not surprising that the majority of GRBs have been detected at intermediate off-axis angles, as visible in Fig. 2 where we show the instrumental positions of the bursts. $23 \%$ of the sample have been detected in the FCFOV, $55 \%$ have been detected with an off-axis angle between $4.5^{\circ}$ and $12^{\circ}$, the remaining $22 \%$ with an angle greater than $12^{\circ}$. Some GRBs were detected at the very edge of the field of view, showing that the IBAS system is able to detect bursts in the whole field of view, provided the signal to noise ratio is large enough.

The GRB positions reported in Table 2 are derived taking into account the most accurate information on the satellite attitude. The errors on these coordinates are in most cases between 1.5 and 3 arcmin, basically limited by the statistical uncertainties. We also report the position of the afterglows (optical or $\mathrm{X}$-ray) when available, as given in the references indicated in the last column. For these bursts, the difference between the ISGRI and the afterglow position is compared with the quoted uncertainties in Fig. 3, where we report the distance between the afterglow position and our position, normalized to the $90 \%$ error radius $R_{90}$ quoted in Table 2, as a function of the off-axis angle. All the positions of the afterglows are within the error regions provided by ISGRI, thus the localization of GRBs are accurate and the error regions are well sized. Moreover there is no evidence of systematic errors depending on the position of the burst on the detector.

Column 13 of Table 2 gives the GRB peak flux in the $15-150 \mathrm{keV}$ energy range integrated over $1 \mathrm{~s}$. This has been computed by using the conversion factor from counts $\mathrm{s}^{-1}$ to photons $\mathrm{cm}^{-2} \mathrm{~s}^{-1}$ derived from the time integrated spectrum of each GRB. This value is corrected for the instrument dead-time.

\subsection{Light curve extraction}

To extract the GRB light curves, as well as for most of our timing analysis, we used the software tools developed for the IBAS 
Table 1. Results of the time-resolved spectral analysis of GRB 060428C. Errors are at 90\% c.l.

\begin{tabular}{|c|c|c|c|c|c|c|c|}
\hline Name & $\begin{array}{l}T_{\text {Start }} \\
\text { UTC }\end{array}$ & $\begin{array}{l}T_{\text {Stop }} \\
\text { UTC }\end{array}$ & Spectral model & Phot. index & $\begin{array}{c}E_{0}(\mathrm{CPL}) \text { or } k T(\mathrm{QT}) \\
\mathrm{keV}\end{array}$ & $\chi_{\text {red }}^{2}$ & d.o.f. \\
\hline \multirow[t]{3}{*}{ Interval 1} & $02: 30: 35$ & $02: 30: 40$ & $\mathrm{PO}$ & $1.9 \pm 0.1$ & & 1.4 & 28 \\
\hline & & & $\mathrm{CPL}$ & $1.3 \pm 0.5$ & $74_{+155}^{-32}$ & 1.2 & 27 \\
\hline & & & QT & $2.3 \pm 0.5$ & $14_{+5}^{+153}$ & 1.2 & 26 \\
\hline \multirow{3}{*}{ Interval 2} & 02:30:40 & $02: 30: 44$ & $\mathrm{PO}$ & $1.46 \pm 0.07$ & & 1.95 & 31 \\
\hline & & & CPL & $0.5 \pm 0.3$ & $64_{+30}^{-16}$ & 1.25 & 30 \\
\hline & & & QT & $1.4 \pm 0.3$ & $17_{+3}^{-3}$ & 1.15 & 29 \\
\hline \multirow[t]{2}{*}{ Interval 3} & $02: 30: 45$ & 02:30:48 & $\mathrm{PO}$ & $2.2 \pm 0.3$ & & 1.29 & 21 \\
\hline & & & $\begin{array}{l}\text { CPL } \\
\text { OT }\end{array}$ & & $\begin{array}{l}\text { Not constrained } \\
\text { Not constrained }\end{array}$ & & \\
\hline
\end{tabular}

interactive quick-look analysis. These tools are more suitable than the standard software (the Off-line Science Analysis software, OSA) for this kind of analysis. The background subtracted light curves, in the 15-300 keV range, are shown in Fig. 12. In order to obtain a better signal to noise ratio, they were extracted only from detector elements where the Pixel Illuminated Fraction (PIF) was greater than 0.5. The background level is estimated with a linear fit to time intervals of different durations appropriately chosen before and after each GRB. The light curves have different bin sizes, appropriate to the counts statistics, and the plotted count rates are normalized taking into account the detector fraction illuminated by the GRBs at the different off-axis angles. All these light curves are corrected for the instrumental dead time, that increased from $\sim 20 \%$ at the beginning of the mission to the current value of $\sim 30 \%$. We have applied this correction via a multiplicative factor computed bin-by-bin, in order to avoid distortions due to dead time variations during the GRBs (for example in the case of GRB 041219 the dead time varied from $25 \%$ to $40 \%$ during the main peaks). Some gaps due to telemetry saturation occurring during the brightest parts of the bursts are visible in the light curves. Unfortunately there is no way to correct for this effect, since no information on the corresponding events is stored onboard or sent to earth. We have used the light curve of each GRB to determine its $t_{90}$, the duration over which $90 \%$ of the fluence of the burst is observed. These intervals are indicated by the dashed lines in Fig. 12, while the dotted lines indicate the time interval from which we extracted the spectrum, when different from $t_{90}$. In Fig. 4 we report the distribution of the durations for the 43 GRBs we have analyzed in this work.

\subsection{Spectral extraction}

In this work we have considered for the spectral analysis only the 45 bursts observed until March of 2007, since the data of the remaining ones are not yet publicly available. For two GRBs (GRB 021125 and GRB 030131) we were not able to perform the analysis (see Sect. 3), so our sample is composed of 43 GRBs. For the imaging and spectral analysis we used the latest release of the standard software (OSA 7.0) distributed by the ISDC. The time integrated spectra of the faintest bursts were extracted from the $t_{90}$ time intervals. For brighter bursts we use longer time intervals, indicated by the dotted lines in Fig. 12. For the spectral extraction we took into account the presence of all the other sources in the field of view detected at more than $5 \sigma$. This is crucial in the analysis of coded mask instruments with a wide field of view, where many detector pixels are exposed to the flux of several sources. Spectra were usually extracted in 64 energy bins between $13 \mathrm{keV}$ and $1 \mathrm{MeV}$, and corrected for the instrumental dead time and for the variation of the mask transparency as a function of the GRB off-axis angle. When the GRB was too weak, or when other bright sources were present in the field of view, we used only 30 energy bins between 13 and $500 \mathrm{keV}$. The count spectra were then fitted using XSPEC v. 11.3 (Arnaud 1996), after excluding the energy bins below $18 \mathrm{keV}$, for which an accurate calibration is not available yet. We have also added a $3 \%$ systematic error to account for the calibration uncertainties. For the GRBs affected by telemetry saturation, we assumed that they kept their average spectrum and flux during the time gaps.

We considered the following spectral models:

- power law (PL) with photon index $\alpha$ :

$$
f(E)=A E^{-\alpha}
$$

- power law with exponential cutoff (CPL):

$$
f(E)=A E^{-\alpha} \mathrm{e}^{-E / E_{0}} ;
$$

- quasi-thermal model (QT) (Ryde 2005; Thompson et al. 2007; Ghirlanda et al. 2007), consisting of a power law and a blackbody:

$$
f(E)=A E^{-\alpha}+B \frac{E^{2}}{(k T)^{4}\left(\mathrm{e}^{\frac{E}{k T}}-1\right)} .
$$

We also performed some fits using the Band model, consisting of two power laws joined with continuity (Band et al. 1993), which is widely used to fit GRB spectra. However, we do not report the results here since these fits to our ISGRI data could not constrain the values of the high-energy photon index. This means in practice that the Band model reduces to the CPL model when used in an energy band as small as ours.

\section{Results}

The spectral results for the power law model are summarized in Table 3. These fits are in general acceptable and give photon index values around $\sim 1.6$ (see Fig. 5). Most of the burst spectra that result in unacceptable fits with this model give better results with the introduction of an exponential cut-off. The resulting best fit parameters are reported in Table 4, where also the F-test results are given to show the significance of the fit improvement. The confidence contours of the photon index and cut-off energy are shown in Fig. 6. Almost all the bursts showing evidence for a curved spectrum in the ISGRI energy range can also be fitted with the QT model, giving the best fit parameters listed in Table 5. 
Table 2. Observed properties of INTEGRAL GRBs. GRBs detected after March 2007 are given here for completeness, but we have only extracted their coordinates and their $t_{90}$.

\begin{tabular}{|c|c|c|c|c|c|c|c|c|c|c|c|c|c|c|}
\hline \# & Name & $\begin{array}{l}\text { Time } \\
\text { (UTC) }\end{array}$ & $\begin{array}{c}\text { RA } \\
\text { (deg) }\end{array}$ & $\begin{array}{c}\text { Dec } \\
(\text { deg })\end{array}$ & $\begin{array}{c}L \\
(\mathrm{deg})\end{array}$ & $\begin{array}{c}B \\
(\mathrm{deg})\end{array}$ & $\begin{array}{l}\text { Pos.error } \\
\text { (arcmin) }\end{array}$ & $\begin{array}{l}\text { After } \\
\text { X O }\end{array}$ & $\begin{array}{l}\text { glow } \\
\text { R }\end{array}$ & $z$ & $\begin{array}{l}T_{90} \\
\text { (s) }\end{array}$ & $\begin{array}{l}T_{50} \\
(\mathrm{~s})\end{array}$ & $\begin{array}{c}\text { Peak Fl. } \\
\left(\mathrm{ph} \mathrm{cm}^{-2} \mathrm{~s}^{-1}\right)\end{array}$ & $\begin{array}{l}\text { Position } \\
\text { reference }\end{array}$ \\
\hline 1 & $021125^{3}$ & $17: 58: 30$ & 296.983 & 28.391 & 64.347 & 1.476 & 2.0 & -- & - & - & 24 & n.a. & $>22$ & - \\
\hline 2 & 021219 & $07: 33: 54$ & 282.613 & 31.950 & 61.88 & 14.14 & 1.14 & - - & - & - & 5 & 1.0 & $>4.5$ & - \\
\hline 3 & $030131^{4}$ & $07: 38: 49$ & 202.086 & 30.673 & 58.809 & 81.183 & 2.1 & $-\mathrm{Y}$ & - & - & 124 & 97 & $>2.1$ & - \\
\hline \multirow[t]{2}{*}{4} & 030227 & $08: 42: 02$ & 74.394 & 20.492 & 180.90 & -13.76 & 1.9 & Y Y & - & - & 15 & 9.0 & 1.4 & \\
\hline & & & 74.387711 & 20.484694 & & & 0.0005 & & & & & & & Opt, GCN 1907 \\
\hline 5 & 030320 & 10:11:52 & 267.892 & -25.330 & 3.79 & 0.71 & 1.4 & -- & - & - & 48 & 33.0 & 10.6 & - \\
\hline 6 & 030501 & 03:10:02 & 286.386 & 6.277 & 40.12 & -0.26 & 1.01 & -- & - & - & 25 & 8.0 & 3.6 & - \\
\hline 7 & 030529 & $19: 53: 18$ & 145.107 & -56.3425 & 278.69 & -2.74 & 3.0 & - - & - & - & 16 & 8.0 & 0.5 & - \\
\hline \multirow[t]{2}{*}{8} & 031203 & $22: 01: 27$ & 120.634 & -39.851 & 255.74 & -4.80 & 1.53 & Y Y & Y & 0.105 & 19 & 8.0 & 2.2 & \\
\hline & & & 120.62579 & -39.85112 & & & 0.012 & & & & & & & X, GCN 2490 \\
\hline \multirow[t]{2}{*}{9} & 040106 & $17: 55: 10$ & 178.042 & -46.797 & 292.50 & 14.88 & 2.23 & Y Y & - & - & 48 & 43.0 & 0.9 & \\
\hline & & & 178.05179 & -46.78775 & & & 0.012 & & & & & & & X, GCN 2510 \\
\hline \multirow[t]{2}{*}{10} & 040223 & $13: 24: 51$ & 249.877 & -41.927 & 341.61 & 3.19 & 0.025 & $\mathrm{Y}-$ & - & - & 198 & 150.0 & 0.45 & \\
\hline & & & 249.87571 & -41.93325 & & & 0.025 & & & & & & & X, GCN 2547 \\
\hline 11 & 040323 & 13:02:58 & 208.469 & -52.354 & 312.52 & 9.36 & 1.26 & $-?$ & - & - & 14 & 6.0 & 1.9 & - \\
\hline 12 & 040403 & 05:08:03 & 115.226 & 68.215 & 147.59 & 29.62 & 1.16 & - - & - & - & 15 & 6.0 & 0.6 & - \\
\hline 13 & 040422 & $06: 57: 59$ & 280.505 & 1.981 & 33.62 & 3.00 & 0.95 & -- & - & - & 4 & 1.8 & 2.8 & - \\
\hline 14 & 040624 & $08: 21: 35$ & 195.030 & -3.583 & 307.18 & 59.21 & 2.8 & -- & - & - & 27 & 10.0 & 0.9 & - \\
\hline 15 & 040730 & 02:12:06 & 238.302 & -56.470 & 326.13 & -2.02 & 1.35 & -- & - & - & 42 & 18.0 & 0.5 & - \\
\hline \multirow[t]{2}{*}{16} & 040812 & $06: 01: 52$ & 246.511 & -44.703 & 337.89 & 3.09 & 1.65 & $\mathrm{Y} ?$ & $?$ & $\left({ }^{5}\right)$ & 8 & 3.0 & 0.7 & \\
\hline & & & 246.52083 & -44.70889 & & & 0.017 & & & & & & & X, GCN 2649 \\
\hline \multirow[t]{2}{*}{17} & 040827 & $11: 50: 50$ & 229.255 & -16.152 & 346.46 & 34.15 & 2.36 & Y Y & - & - & 32 & 12.0 & 1.0 & \\
\hline & & & 229.25558 & -16.14142 & & & 0.003 & & & & & & & $\mathrm{NIR}^{6}$ \\
\hline 18 & 040903 & $18: 17: 58$ & 270.824 & -25.258 & 5.14 & -1.52 & 3.20 & -- & - & - & 7 & 3.0 & 0.42 & - \\
\hline 19 & 041015 & $11: 11: 33$ & 4.690 & 66.853 & 119.71 & 4.19 & 2.81 & - & - & - & 30 & 12.0 & 0.4 & - \\
\hline \multirow[t]{2}{*}{20} & 041218 & $15: 45: 44$ & 24.794 & 71.345 & 126.77 & 8.82 & 0.86 & $-\mathrm{Y}$ & - & - & 38 & 27.3 & 3.8 & \\
\hline & & & 24.78167 & 71.34167 & & & 0.025 & & & & & & & X, GCN 2861 \\
\hline \multirow[t]{2}{*}{21} & 041219 & $01: 42: 13$ & 6.1143 & 62.839 & 119.86 & 0.13 & 0.34 & $-\mathrm{Y}$ & $\mathrm{Y}$ & - & 239 & 129 & $>17.2$ & \\
\hline & & & 6.1153330 & 62.842640 & & & 0.003 & & & & $(460)^{7}$ & $(152)^{6}$ & & Opt, GCN 2893 \\
\hline 22 & 050129 & 20:03:05 & 252.789 & -3.097 & 15.23 & 24.88 & 2.32 & -- & - & - & 30 & 12.0 & 0.4 & - \\
\hline \multirow[t]{2}{*}{23} & 050223 & 03:09:00 & 271.398 & -62.459 & 331.52 & -18.82 & 4.46 & $\mathrm{Y}-$ & - & 0.591 & 30 & 12.0 & 0.9 & \\
\hline & & & 271.38538 & -62.472519 & & & 0.025 & & & & & & & X, GCN 3109 \\
\hline \multirow[t]{2}{*}{24} & 050502 & 02:14:01 & 202.436752 & 42.671132 & 98.76 & 72.61 & 0.62 & $-\mathrm{Y}$ & - & 3.793 & $>11$ & $>6.0$ & 2.4 & \\
\hline & & & 202.44292 & 42.674362 & & & n.d. & & & & & & & Opt, GCN 3322 \\
\hline \multirow[t]{2}{*}{25} & 050504 & 08:00:50 & 200.998 & 40.697 & 98.66 & 74.85 & 0.80 & $\mathrm{Y}-$ & - & - & 44 & 22.0 & 0.5 & \\
\hline & & & 201.0054 & 40.70333 & & & 0.11 & & & & & & & X, GCN 3359 \\
\hline 26 & 050520 & $00: 05: 57$ & 192.511 & 30.450 & 127.88 & 86.66 & 1.16 & $\mathrm{Y}-$ & - & - & 52 & 30.8 & 1.2 & \\
\hline & & & 192.52583 & 30.450556 & & & 0.08 & & & & & & & X, GCN 3343 \\
\hline 27 & 050522 & 06:00:21 & 200.137 & 24.793 & 14.90 & 83.04 & 3.0 & $?-$ & - & - & 11 & 2.7 & 0.3 & - \\
\hline 28 & 050525 & $00: 02: 53$ & 278.117 & 26.332 & 54.95 & 15.54 & 1.4 & Y Y & - & 0.606 & 9 & 6.0 & $>56.2$ & \\
\hline & & & 278.13571 & 26.339582 & & & 0.002 & & & & & & & Opt, GCN 3493 \\
\hline 29 & 050626 & 03:46:07 & 186.741 & -63.137 & 300.18 & -0.38 & 1.35 & -- & - & - & 56 & 24.0 & 0.4 & - \\
\hline 30 & 050714 & $00: 05: 53$ & 43.612 & 69.120 & 133.56 & 8.82 & 1.46 & $\mathrm{Y} ?$ & - & - & 34 & 24.0 & 0.4 & \\
\hline & & & 43.592499 & 69.112778 & & & 0.115 & & & & & & & X, GCN 3649 \\
\hline 31 & 050918 & $15: 36: 38$ & 267.650 & -25.406 & 3.57 & 0.91 & 3.33 & $?-$ & - & - & 280 & 226.0 & 2.4 & - \\
\hline 32 & 050922 & $13: 43: 20$ & 271.094 & -32.041 & 359.39 & -5.09 & 2.9 & -- & - & - & 10 & 10.0 & 0.17 & - \\
\hline 33 & 051105B & $11: 05: 41$ & 9.468 & -40.479 & 313.95 & -76.35 & 1.1 & -- & - & - & 14 & 6.0 & 0.4 & - \\
\hline 34 & 051211B & 22:06:07 & 345.667 & 55.081 & 107.72 & -4.55 & 1.34 & Y Y & $?$ & - & 47 & 22.0 & 1.0 & \\
\hline & & & 345.67322 & 55.080971 & & & 0.006 & & & & & & & Opt, GCN 4358 \\
\hline 35 & 060114 & $12: 39: 31$ & 195.276 & -4.735 & 307.54 & 58.04 & 1.47 & -- & - & - & 80 & 32.0 & 0.3 & - \\
\hline 36 & 060130 & $04: 56: 29$ & 229.248 & -36.921 & 332.72 & 17.41 & 2.0 & -- & - & - & 19 & 7.0 & 0.25 & - \\
\hline 37 & 060204 & $13: 19: 39$ & 232.254 & -39.467 & 333.25 & 13.96 & 1.7 & -- & - & - & 52 & 30.0 & 0.3 & - \\
\hline 38 & $060428 \mathrm{C}$ & $02: 30: 35$ & 285.227 & -9.556 & 25.46 & -6.43 & 0.72 & -- & - & - & 10 & 4.8 & 4.9 & - \\
\hline 39 & 060901 & $18: 43: 55$ & 287.164 & -6.639 & 28.95 & -6.83 & 1.5 & $\mathrm{Y} ?$ & - & - & 16 & 3.2 & 11.4 & \\
\hline & & & 287.15805 & -6.6394440 & & & 0.07 & & & & & & & X, GCN 5496 \\
\hline 40 & 060912B & $17: 32: 11$ & 271.203 & -19.872 & 10.01 & 0.76 & 2.6 & -- & - & - & 140 & 60.0 & 0.1 & - \\
\hline 41 & 060930 & 09:04:12 & 304.511 & -23.635 & 19.56 & -28.94 & 2.3 & -- & - & - & 9 & 4.0 & 0.4 & - \\
\hline 42 & 061025 & $18: 35: 53$ & 300.904 & -48.244 & 350.95 & -31.58 & 0.87 & Y Y & - & - & 11 & 4.0 & 1.6 & \\
\hline & & & 300.91177 & -48.242981 & & & 0.008 & & & & & & & Opt, GCN 5754 \\
\hline 43 & 061122 & $07: 56: 50$ & 303.836 & 15.511 & 56.65 & -10.66 & 0.50 & Y Y & - & - & 12 & 4.9 & $>33.0$ & \\
\hline & & & 303.83267 & 15.517361 & & & 0.008 & & & & & & & Opt, GCN 5849 \\
\hline
\end{tabular}

${ }^{3}$ Observed during commissioning, Malaguti et al. (2003); ${ }^{4}$ Observed during a slew, Gotz et al. (2003); ${ }^{5}$ D'Avanzo et al. (2006) derived a photometric redshift $0.3 \leq z \leq 0.7 ;{ }^{6}$ de Luca et al. (2005); ${ }^{7}$ if we include precursors, see text. 
Table 2. continued.

\begin{tabular}{|c|c|c|c|c|c|c|c|c|c|c|c|c|c|c|}
\hline \# & Name & $\begin{array}{l}\text { Time } \\
\text { (UTC) }\end{array}$ & $\begin{array}{c}\text { RA } \\
(\text { deg }) \\
\end{array}$ & $\begin{array}{l}\begin{array}{c}\text { Dec } \\
(\mathrm{deg})\end{array} \\
\end{array}$ & $\begin{array}{c} \\
(\mathrm{deg}) \\
\end{array}$ & $\begin{array}{c}B \\
(\mathrm{deg}) \\
\end{array}$ & $\begin{array}{l}\text { Pos.error } \\
(\operatorname{arcmin})\end{array}$ & $\begin{array}{l}\text { After } \\
\text { X O }\end{array}$ & $\begin{array}{l}\text { slow } \\
\mathrm{R}\end{array}$ & $z$ & $\begin{array}{l}T_{90} \\
(\mathrm{~s}) \\
\end{array}$ & $\begin{array}{l}T_{50} \\
(\mathrm{~s}) \\
\end{array}$ & $\begin{array}{c}\text { Peak Fl. } \\
\left(\mathrm{ph} \mathrm{cm}^{-2} \mathrm{~s}^{-1}\right)\end{array}$ & $\begin{array}{l}\text { Position } \\
\text { reference }\end{array}$ \\
\hline 44 & 070309 & 10:00:39 & $\begin{array}{c}263.680 \\
263.6629\end{array}$ & $\begin{array}{c}-37.9525 \\
-37.93006\end{array}$ & 351.16 & -2.83 & $\begin{array}{c}2.1 \\
0.08\end{array}$ & $\mathrm{Y}-$ & - & - & 22 & 12.0 & 0.4 & X, GCN 6187 \\
\hline 45 & 070311 & 01:52:34 & $\begin{array}{l}87.543158 \\
87.534210\end{array}$ & $\begin{array}{c}3.39136 \\
3.3750800\end{array}$ & 202.76 & -12.00 & $\begin{array}{l}2.04 \\
0.02\end{array}$ & Y Y & - & - & 32 & 16.0 & 1.1 & X, GCN 6190 \\
\hline 46 & 070615 & $02: 20: 37$ & 44.316 & -4.410 & 181.41 & -52.36 & 1.92 & $?-$ & - & - & 15 & 7.0 & n.a. & - \\
\hline 47 & 070707 & 16:08:38 & $\begin{array}{c}267.738 \\
267.74396\end{array}$ & $\begin{array}{c}-68.924 \\
-68.924225\end{array}$ & 324.61 & -20.00 & $\begin{array}{c}1.85 \\
0.008\end{array}$ & Y Y & - & - & 0.7 & 0.3 & n.a. & X, GCN 6612 \\
\hline 48 & 070925 & $15: 52: 32$ & $\begin{array}{c}253.214 \\
253.21699\end{array}$ & $\begin{array}{c}-22.036 \\
-22.028561\end{array}$ & 358.96 & 13.71 & $\begin{array}{l}0.1 \\
0.1\end{array}$ & $\mathrm{Y}-$ & - & - & 19 & 7.0 & n.a. & X, GCN 6826 \\
\hline 49 & 071017 & 00:58:08 & 274.706 & -15.968 & 15.03 & -0.27 & 2.43 & -- & - & - & 0.5 & 0.2 & n.a. & - \\
\hline 50 & 071109 & $20: 35: 55$ & 289.9 & & 37.99 & -5.33 & 2. & -- & - & - & 30 & n.a. & n.a & - \\
\hline 51 & 080120 & $17: 28: 28$ & $\begin{array}{c}225.254 \\
225.2636\end{array}$ & $\begin{array}{c}-10.885 \\
-10.8749\end{array}$ & 346.86 & 40.68 & $\begin{array}{c}2.0 \\
0.01\end{array}$ & Y Y & - & - & 15 & n.a. & n.a. & Opt, GCN 7198 \\
\hline 52 & 080414 & $22: 33: 30$ & 272.133 & -18.829 & 11.36 & 0.55 & 2.0 & -- & - & - & 8 & n.a. & n.a. & - \\
\hline 53 & 080603 & $11: 18: 15$ & $\begin{array}{c}279.408 \\
279.4082\end{array}$ & $\begin{array}{c}62.735 \\
62.7441\end{array}$ & 92.50 & 25.46 & $\begin{array}{c}2.0 \\
0.01\end{array}$ & Y Y & - & - & 150 & n.a. & n.a. & Opt, GCN 7976 \\
\hline 54 & 080613 & $09: 35: 21$ & $\begin{array}{c}213.275 \\
213.2709\end{array}$ & $\begin{array}{c}5.169 \\
5.1732\end{array}$ & 348.09 & 60.66 & $\begin{array}{c}1.5 \\
0.01\end{array}$ & Y Y & - & - & 30 & n.a. & n.a. & Opt, GCN 7872 \\
\hline 55 & 080723B & $\begin{array}{l}13: 22: 15 \\
11 \cdot 02 \cdot 36\end{array}$ & 176.833 & -60.245 & 295.05 & 1.64 & 1.5 & $\begin{array}{l}-- \\
--\end{array}$ & - & - & 95 & n.a. & n.a. & - \\
\hline 56 & 080922 & 11:03:36 & 270.837 & -22.509 & 7.56 & -0.19 & 1.5 & -- & - & - & 60 & n.a. & n.a. & - \\
\hline
\end{tabular}

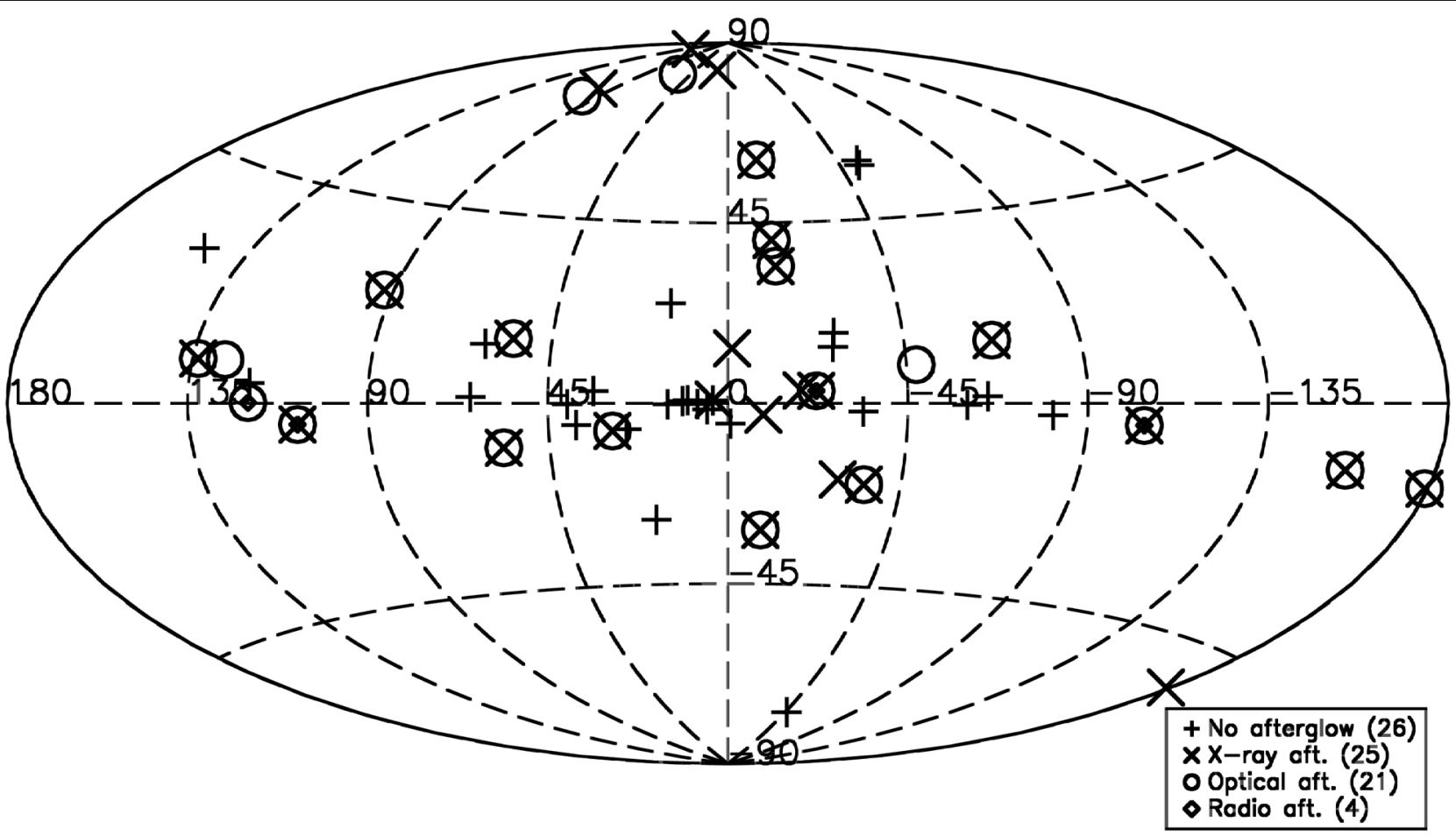

Fig. 1. Sky positions of the burst detected by IBAS in Galactic coordinates.

\subsection{GRB 021125}

This burst, the first one discovered by IBIS, was observed during the INTEGRAL Performance and Verification phase, while the instruments were not yet fully operational. In particular, during the observation of this burst, ISGRI could transmit to ground only a limited amount of information because the majority of the telemetry allocation was used for other data. For this reason, the ISGRI data on GRB 021125 cannot be easily analyzed with the standard software and require an ad hoc treatment, as reported in Malaguti et al. (2003). We have not considered this burst in our work, but, for completeness, we give in Table 2 the results obtained by these authors.

\subsection{GRB 021219}

The results of this GRB, the first to be detected and localized by the IBAS software, were first reported by Mereghetti et al. (2003a). By comparison with the Crab, due to the lack at that time of a proper calibration, they found a power law photon in$\operatorname{dex} \alpha=2$ for the average spectrum, with evidence for a hard-tosoft evolution. We obtain a consistent value for the fluence, but a slightly harder spectrum (see Table 3). Moreover, thanks to the improved spectral calibration now available, we have found convincing evidence of a spectral break at $E_{0}=50_{+66}^{-11} \mathrm{keV}$. Indeed the fit with the $\mathrm{CP}$ model results in a significant improvement, with an F-test chance probability of only $4 \times 10^{-4}$ (see Table 4). 


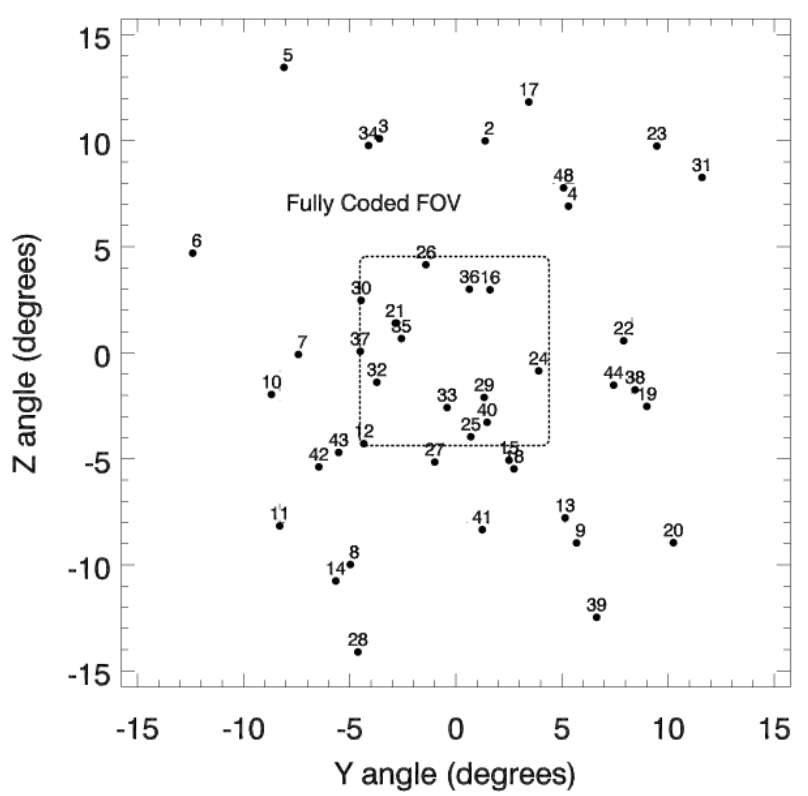

Fig. 2. GRB positions in the detector coordinates. The region inside the dotted rectangle is the fully coded field of view.

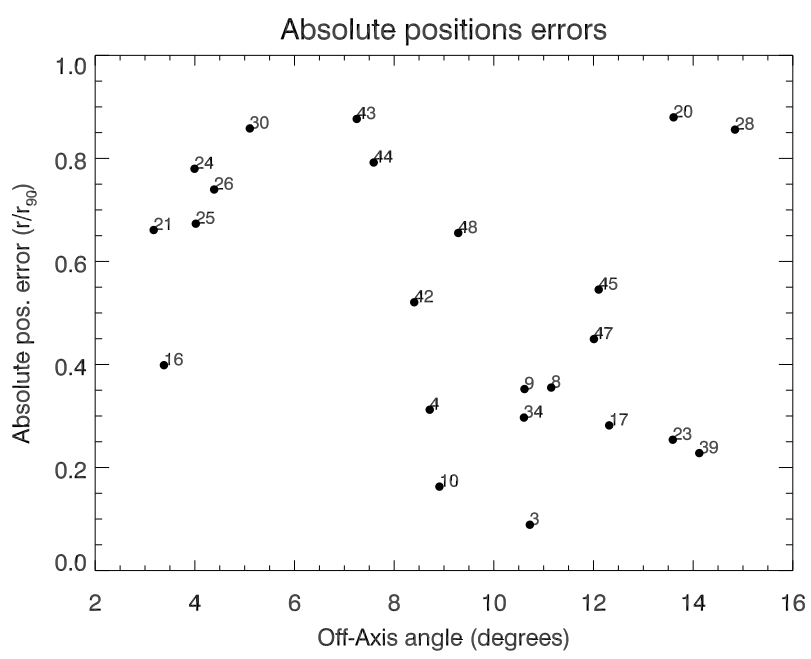

Fig. 3. Absolute position errors $r$ as a function of the off-axis angle, for bursts with a detected optical afterglow. $r$ is defined as the difference between the position obtained with ISGRI data and that of the optical afterglow. The error on the position of the optical afterglows is negligible. The absolute position error is given in units of $r_{90}$, that is, the $90 \%$ error radius estimated during the analysis of ISGRI data. All the afterglows were found inside the error circle provided by ISGRI.

We confirm the spectral evolution, as shown by the hardness ratio reported in Fig. 7.

\subsection{GRB 030131}

This burst, lasting about two minutes, was partly observed during a satellite slew. The slew started at 07:39:09 UT, $20 \mathrm{~s}$ after the beginning of the burst, and resulted in a variation of $\sim 2^{\circ}$ of the GRB position in the ISGRI field of view during the source activity period. The OSA software cannot be used to analyze data collected during slews, so we could not analize this GRB. We refer to the work of Götz et al. (2003) for a proper analysis using the IBAS software.

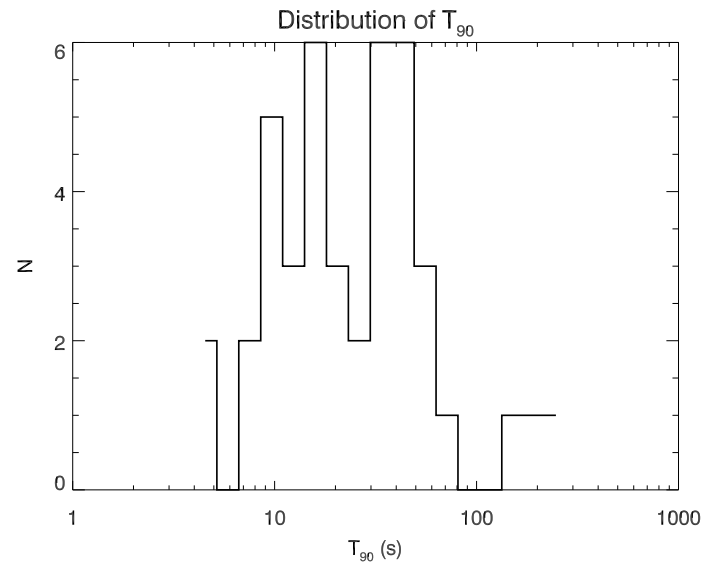

Fig. 4. Distribution of $t_{90}$ for INTEGRAL GRBs we have analyzed in this work. No short bursts $\left(t_{90}<2 \mathrm{~s}\right)$ are present in the sample.

\subsection{GRB 030227}

This is the first INTEGRAL GRB for which X-ray and optical afterglow searches were successful (Mereghetti et al. 2003c; Castro-Tirado et al. 2003). Evidence for intrinsic absorption, as well as possible emission lines, were reported in the spectrum of its X-ray afterglow (Mereghetti et al. 2003c; Watson et al. 2003). The ISGRI spectrum reported here is slightly harder, but still consistent with that derived by Mereghetti et al. (2003c) using preliminary calibrations.

\subsection{GRB 030320}

This burst shows three different emission episodes, with several peaks. The average spectrum is poorly fitted by a power law $\left(\chi_{\text {red }}^{2}=1.8\right.$ with 24 d.o.f.), and also the CP (or Band) model and the QT model do not give better fits. On the contrary, separately fitting the three emission episodes, we found that each of them is well described by a power law. The corresponding photon indexes are $1.27 \pm 0.08$ ( $\chi_{\text {red }}^{2}=1.39$ with 39 d.o.f. $), 2.01 \pm 0.3$ $\left(\chi_{\text {red }}^{2}=1.41\right.$ with 34 d.o.f. $)$ and $1.49 \pm 0.1\left(\chi_{\text {red }}^{2}=1.09\right.$ with 24 d.o.f.). The first peak is the hardest, then there is a second softer peak, and a third hard peak again. A similarly complex spectral evolution has been seen in a few other GRBs, for example GRB 060124 (Romano et al. 2006). Foley et al. (2008) reported a time dependent spectral lag: $0.33 \pm 0.03 \mathrm{~s}$ for the first peak, and a value close to zero $(0.05 \pm 0.03 \mathrm{~s})$ for the third one. No spectral lag could be measured for the second peak, because it is observed only in the softer band. We can speculate, in the context of the fireball model, that the second peak could be the onset of the afterglow, i.e. it could be due to the external shock caused by the contact between the circumstellar medium and the expanding fireball, resulting from the merging of the two shells that produced the first peak. The time between the onset of the first peak and the onset of the second one $(\Delta t \simeq 30 \mathrm{~s})$ is compatible with this hypothesis, provided we are in the thin shell case (Sari \& Piran 1999), as expected when the prompt and the afterglow phases are distinct: using a typical Lorentz factor for the expanding shell $(\Gamma \sim 100)$, the start of the emission from the external shock would have taken place at the deceleration radius $R=2 \Gamma^{2} c \Delta t \simeq 1.8 \times 10^{16} \mathrm{~cm}$ from the center, that is a typical value in this scenario (see for example Piran 1999).

Our spectral results on GRB 030320 differ from those reported by von Kienlin et al. (2003), who found systematically 
Table 3. Results of fitting spectra of INTEGRAL GRBs with a power law model.

\begin{tabular}{|c|c|c|c|c|c|c|c|}
\hline \# & Name & $\begin{array}{c}\text { Peak flux } \\
(15-150 \mathrm{keV}) \\
{\left[10^{-8} \mathrm{erg} / \mathrm{cm}^{2} \mathrm{~s}\right]} \\
\end{array}$ & $\begin{array}{c}\text { Fluence } \\
(20-200 \mathrm{keV}) \\
{\left[10^{-8} \mathrm{erg} / \mathrm{cm}^{2}\right]} \\
\end{array}$ & $\begin{array}{l}\text { Photon index } \\
\text { (power law }\end{array}$ & $\begin{array}{l}\chi_{\text {red }}^{2} \\
\text { model) }\end{array}$ & d.o.f. & $\begin{array}{l}T_{90} \\
{[\mathrm{~s}]} \\
\end{array}$ \\
\hline 2 & 021219 & $>28.7$ & $>94.6$ & $1.69_{+0.09}^{-0.08}$ & 1.46 & 46 & 5.0 \\
\hline 4 & 030227 & 7.0 & 74.9 & $1.75_{+0.11}^{-0.10}$ & 1.0 & 46 & 15.0 \\
\hline 5 & 030320 & 68.0 & 1131.9 & $1.28_{+0.07}^{-0.07}$ & 1.8 & 24 & 48.0 \\
\hline 6 & 030501 & 18.4 & 282.1 & $1.83_{+0.08}^{-0.07}$ & 1.5 & 46 & 25.0 \\
\hline 7 & 030529 & 1.1 & 5.2 & $3.50_{+0.51}^{-0.43}$ & 1.0 & 9 & 16.0 \\
\hline 8 & 031203 & 12.8 & 134.8 & $1.50_{+0.09}^{-0.09}$ & 1.3 & 46 & 19.0 \\
\hline 9 & 040106 & 6.2 & 105.9 & $1.58_{+0.15}^{-0.14}$ & 1.1 & 46 & 48.0 \\
\hline 10 & 040223 & 2.1 & 147.7 & $2.11_{+0.17}^{-0.16}$ & 0.8 & 46 & 198.0 \\
\hline 11 & 040323 & 17.15 & 190.4 & $1.07_{+0.07}^{-0.06}$ & 1.7 & 46 & 14.0 \\
\hline 12 & 040403 & 3.3 & 28.5 & $1.84_{+0.16}^{-0.15}$ & 1.0 & 46 & 15.0 \\
\hline 13 & 040422 & 20.7 & 44.2 & $2.01_{+0.09}^{-0.08}$ & 1.7 & 46 & 4.0 \\
\hline 14 & 040624 & 4.5 & 48.1 & $2.02_{+0.20}^{-0.18}$ & 0.8 & 46 & 27.0 \\
\hline 15 & 040730 & 3.0 & 57.8 & $1.44_{+0.16}^{-0.15}$ & 0.8 & 39 & 42.0 \\
\hline 16 & 040812 & 4.55 & 14.0 & $2.20_{+0.22}^{-0.21}$ & 0.9 & 28 & 8.0 \\
\hline 17 & 040827 & 6.35 & 101.0 & $1.58_{+0.21}^{-0.19}$ & 1.1 & 15 & 32.0 \\
\hline 18 & 040903 & 2.3 & 9.6 & $2.90_{+0.46}^{-0.39}$ & 0.5 & 23 & 7.0 \\
\hline 19 & 041015 & 2.3 & 51.2 & $1.13_{+0.18}^{-0.18}$ & 1.0 & 35 & 30.0 \\
\hline 20 & 041218 & 22.7 & 491.9 & $1.57_{+0.05}^{-0.05}$ & 1.3 & 46 & 38.5 \\
\hline 21 & 041219 & $>130$ & $>2100$ & $1.89_{+0.01}^{0.01}$ & 1.1 & 46 & $239.0(460)^{8}$ \\
\hline 22 & 050129 & 2.4 & 41.0 & $1.79_{+0.27}^{-0.25}$ & 0.7 & 27 & 30.0 \\
\hline 23 & 050223 & 4.0 & 81.5 & $1.64_{+0.24}^{-0.22}$ & 1.0 & 46 & 30.0 \\
\hline 24 & 050502 & 12.3 & $>108.6$ & $1.51_{+0.06}^{-0.05}$ & 1.6 & 46 & $>11.0$ \\
\hline 25 & 050504 & 3.8 & 116.0 & $1.20_{+0.09}^{-0.09}$ & 1.5 & 46 & 44.0 \\
\hline 26 & 050520 & 8.9 & 159.9 & $1.64_{+0.07}^{-0.06}$ & 1.1 & 46 & 52.5 \\
\hline 27 & 050522 & 1.5 & 6.9 & $2.65_{+0.59}^{-0.48}$ & 1.0 & 21 & 10.8 \\
\hline 28 & 050525 & $>314.8$ & $>1300.0$ & $1.93_{+0.00}^{-0.05}$ & 1.9 & 39 & 9.0 \\
\hline 29 & 050626 & 2.7 & 66.5 & $2.04_{+0.15}^{-0.14}$ & 0.6 & 39 & 56.0 \\
\hline 30 & 050714 & 2.9 & 42.7 & $2.03_{+0.21}^{-0.19}$ & 1.0 & 43 & 34.0 \\
\hline 31 & 050918 & 15.3 & 480.0 & $1.77_{+0.10}^{-0.09}$ & 0.9 & 39 & 280.0 \\
\hline 32 & 050922 & 1.1 & 5.9 & $1.85_{+0.69}^{-0.58}$ & 0.7 & 11 & 10.0 \\
\hline 33 & 051105B & 3.6 & 20.4 & $1.84_{+0.26}^{-0.23}$ & 1.0 & 46 & 14.0 \\
\hline 34 & 051211B & 7.0 & 179.0 & $1.54_{+0.10}^{-0.09}$ & 1.0 & 39 & 47.0 \\
\hline 35 & 060114 & 1.6 & 98.9 & $0.95_{+0.19}^{-0.18}$ & 1.1 & 21 & 80.0 \\
\hline 36 & 060130 & 1.9 & 22.5 & $1.59_{+0.34}^{-0.31}$ & 1.4 & 22 & 19.0 \\
\hline 37 & 060204 & 1.8 & 46.8 & $1.35_{+0.24}^{-0.23}$ & 1.0 & 25 & 52.0 \\
\hline 38 & $060428 \mathrm{C}$ & 30.1 & 201.0 & $1.55_{+0.05}^{-0.04}$ & 1.9 & 46 & 10.4 \\
\hline 39 & 060901 & 69.2 & 564.6 & $1.43_{+0.06}^{-0.06}$ & 1.1 & 46 & 16.0 \\
\hline 40 & 060912B & 1.3 & 69.4 & $1.65_{+0.30}^{-0.28}$ & 1.2 & 25 & 140.0 \\
\hline 41 & 060930 & 4.9 & 26.3 & $1.51_{+0.30}^{-0.27}$ & 1.2 & 33 & 9.0 \\
\hline 42 & 061025 & 9.7 & 97.5 & $1.34_{+0.08}^{-0.07}$ & 1.6 & 46 & 11.0 \\
\hline 43 & 061122 & 146.2 & 459.2 & $1.71_{+0.05}^{-0.04}$ & 1.6 & 39 & 11.9 \\
\hline 44 & 070309 & 2.6 & 35.7 & $1.36_{+0.31}^{-0.30}$ & 1.3 & 16 & 22.0 \\
\hline 45 & 070311 & 8.5 & 137.9 & $1.34_{+0.13}^{-0.12}$ & 1.0 & 46 & 32.0 \\
\hline
\end{tabular}

\footnotetext{
${ }^{8}$ If we include the precursors, see text.
} 
Table 4. Results of fitting spectra of some INTEGRAL GRBs with a cut-off power law model.

\begin{tabular}{lccccccc}
\hline \hline$\#$ & Name & $\begin{array}{c}\text { Fluence } \\
(20-200 \mathrm{keV}) \\
{\left[10^{-8} \mathrm{erg} / \mathrm{cm}^{2}\right]}\end{array}$ & Photon index & $\begin{array}{c}\text { Cut-off power law model } \\
\text { Cutoff energy } E_{0}\end{array}$ & $\chi_{\text {red }}^{2}$ & d.o.f. & probability \\
\hline 2 & 021219 & $>83.4$ & $0.67_{+0.59}^{-0.30}$ & $50_{+69}^{-11}$ & 1.1 & 45 & $4 \times 10^{-4}$ \\
11 & 040323 & 189.9 & $0.44_{+0.26}^{-0.29}$ & $120_{+84}^{-39}$ & 1.3 & 45 & $2 \times 10^{-4}$ \\
13 & 040422 & 38.9 & $0.89_{+0.40}^{-0.44}$ & $45_{+25}^{-13}$ & 1.1 & 45 & $1 \times 10^{-5}$ \\
24 & 050502 & 101.2 & $0.86_{+0.22}^{-0.24}$ & $91_{+47}^{-25}$ & 1.0 & 45 & $3 \times 10^{-6}$ \\
25 & 050504 & 104.4 & $0.28_{+0.42}^{-0.48}$ & $69_{+60}^{-24}$ & 1.2 & 45 & $6 \times 10^{-4}$ \\
28 & 050525 & $>1027.7$ & $1.30_{+0.21}^{-0.22}$ & $83_{+40}^{-22}$ & 1.2 & 38 & $2 \times 10^{-6}$ \\
38 & $060428 \mathrm{C}$ & 185.9 & $0.86_{+0.20}^{-0.20}$ & $85_{+34}^{-19}$ & 0.9 & 45 & $2 \times 10^{-8}$ \\
42 & 061025 & 88.9 & $0.41_{+0.35}^{-0.39}$ & $66_{+40}^{-20}$ & 1.1 & 45 & $1 \times 10^{-5}$ \\
43 & 061122 & 438.6 & $1.24_{+0.16}^{-0.16}$ & $122_{+60}^{-31}$ & 0.8 & 45 & $2 \times 10^{-7}$ \\
\hline
\end{tabular}

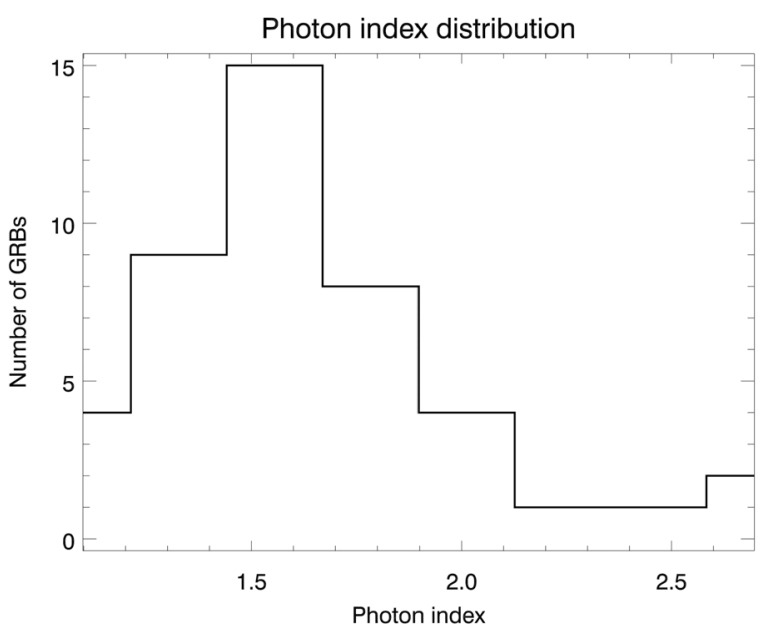

Fig. 5. The distribution of the photon index of the single power law fits. All but two spectra are well described by a power law and are given in this plot, although for 9 of them a CPL or a QT model give a better fit.

softer spectra. This is probably due to the inadequacy of the preliminary calibrations they used for this burst at a large off-axis angle $\left(15.7^{\circ}\right)$.

\subsection{GRB 030501}

This single-peaked GRB was seen in the partially coded field of view, at $\sim 13^{\circ}$ from the instrument axis. Due to the very large off-axis angle, only $10 \%$ of the detector plane was illuminated by the source. The spectrum of GRB 030501 is well fitted by a power law, with no evidence for spectral cutoff or spectral evolution. While these could be intrinsic properties of this burst, it is also likely that they are simply due to the poor statistics of the data. Our results are fully compatible with those reported by Beckmann et al. (2003).

\subsection{GRB 030529}

GRB 030529 was discovered during an off-line re-analysis of the INTEGRAL data, performed with an updated version of the IBAS tools, more sensitive than those used in real time during the first months of the mission. GRB 030529 was a 20 s long, faint burst, that occurred during a period of highly variable instrumental background (see Fig. 12). This explains why it was missed by the first version of the IBAS system. The spectrum we extracted is well fitted by a power law with a very soft photon index $\alpha=3.5^{-0.43}$. Thus GRB 030529 belongs to the class of X-ray Flashes (XRF).

\subsection{GRB 031203}

GRB 031203 is among the most interesting GRBs discovered by INTEGRAL. Its afterglow was observed at X-ray (Watson et al. 2004), IR/Optical (Cobb et al. 2004), and radio (Frail 2003) wavelengths, and spectroscopic evidence of an associated type Ic Supernova was found (Malesani et al. 2004b). The discovery of its host galaxy led to a redshift determination of $z=0.106$ (Prochaska et al. 2004), implying a surprisingly small isotropicequivalent energy $E_{\text {iso }}=(6-14) \times 10^{49} \mathrm{erg}$. This value and the measure of $E_{\text {peak }}=151 \pm 50 \mathrm{keV}$, derived from the average spectrum as observed by Konus-Wind (Ulanov et al. 2005), make GRB 031203 an outlier in the $E_{\text {peak }}-E_{\text {iso }}$ relation (Amati 2006).

The X-ray images obtained with XMM-Newton led to the discovery of expanding rings due to the scattering of the GRB X-ray emission by dust grains in our Galaxy (Vaughan et al. 2004). The modelling of this GRB "echo" provides an indirect means to estimate the intensity of the prompt GRB emission at X-ray energies. This gives some evidence for an X-ray flux component in excess of the low-energy extrapolation of the INTEGRAL spectrum (Vaughan et al. 2004; Tiengo \& Mereghetti 2006). Taking into account this phenomenon and other effects, GRB 031203 could be made consistent with the Amati correlation (Ghisellini et al. 2006).

The spectrum we extracted is well described by a power law. No improvements are achieved by using more complicated models, and we found no evidence of a spectral break. Our spectral parameters basically confirm the results given by Sazonov et al. (2004): their values for the fluence $\left(2.0 \times 10^{-6} \mathrm{erg} \mathrm{cm}^{-2}\right)$ and for the photon index $(\alpha=1.63 \pm 0.06)$ are slightly different from ours, but this differences could easily be due to the much better calibration we used. Using Konus-Wind data, Ulanov et al. (2005) found a cutoff around $150 \mathrm{keV}$. We can obtain only a lower limit on the cutoff $\left(E_{0}>100 \mathrm{keV}, 90 \%\right.$ c.l. $)$, because the fluence at high energy is too small. 

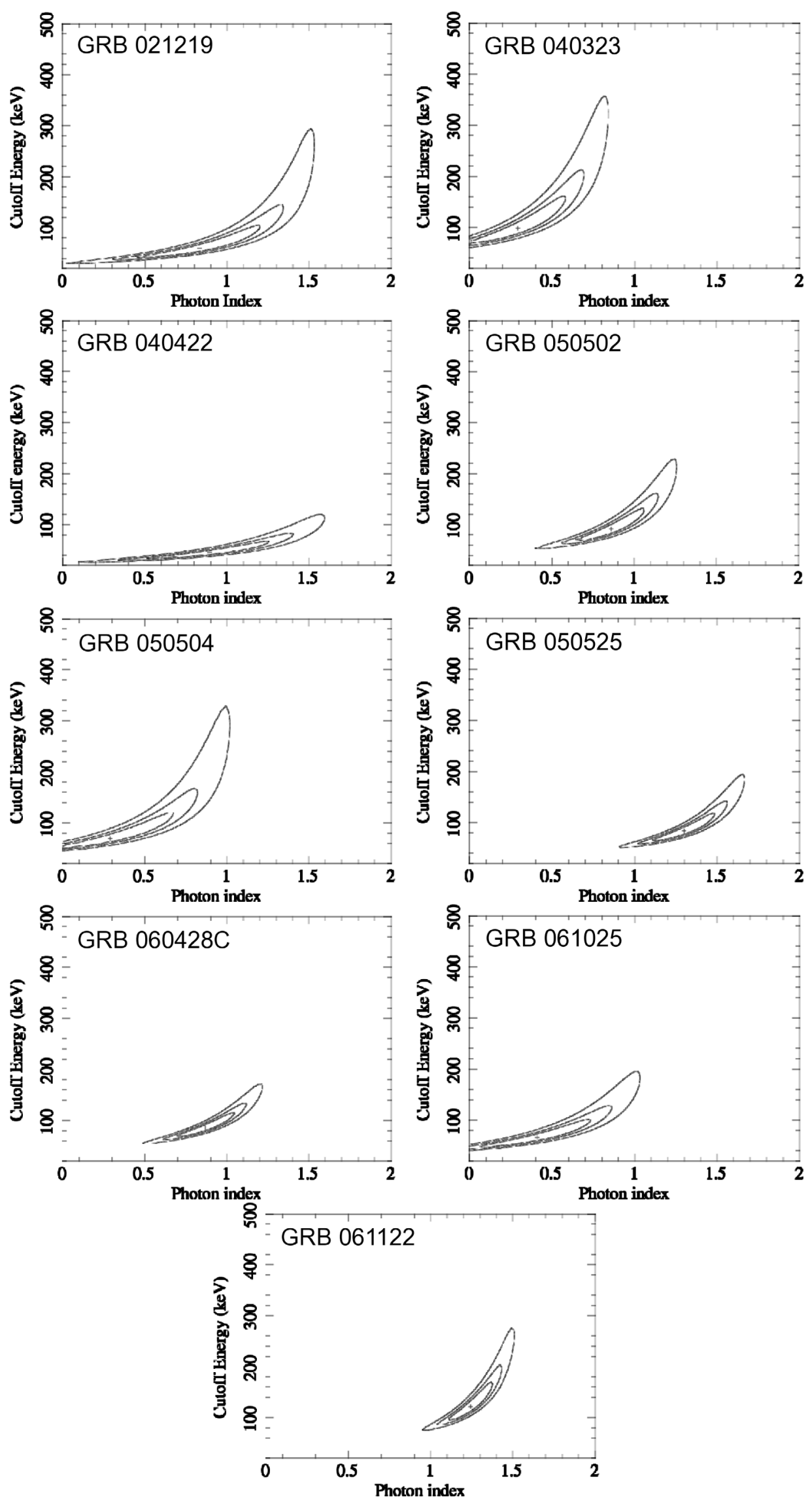

Fig. 6. Contour plot of the parameters of the CPL model. The lines are respectively $68 \%, 90 \%$ and $99 \%$ confidence levels. To make comparisons easier the axes are the same in all plots. 


\subsection{GRB 040106}

This GRB is composed of two peaks separated by a quiescent period of $30 \mathrm{~s}$. To optimize the signal to noise ratio, we extracted the average spectrum removing the quiescent period. The best fit model is a power law: no cutoff is detected. Our photon index is a little harder (but compatible) with that reported by Moran et al. (2005).

We found evidence of spectral evolution between the two peaks. Their spectra are well described by a power law, with a photon index respectively of $\alpha=1.80 \pm 0.14\left(\chi_{\text {red }}^{2}=1.05\right.$ with 46 d.o.f.) and $\alpha=1.41 \pm 0.17$ ( $\chi_{\text {red }}^{2}=1.35$ with 46 d.o.f.). This soft-to-hard evolution, also noted by Moran et al. (2005), is not usual. We note however that the quiescent time between the two peaks is quite long compared to the duration of the peaks, so that the two emission episodes are distinct and in principle could be uncorrelated. This is not the only peculiarity of this GRB: the $\mathrm{X}$-ray afterglow, promptly observed with $X M M$-Newton, decays as expected like a power law with an index of $1.46 \pm 0.04$, but is spectrally harder than usual. This behavior is difficult to describe with a normal fireball model, and requires ad-hoc adjustments (Moran et al. 2005).

\subsection{GRB 040223}

This faint GRB occurred in the Galactic plane $\left(b=3.2^{\circ}\right)$ at only $19^{\circ}$ from the Galactic center, in a region where there are several bright sources. Three low-mass X-ray binary systems were detected by ISGRI with a significance above $5 \sigma$ in the time interval of the GRB, namely 4U 1636-536, H 1705-440, and GX 340+0. The presence of these sources has been properly taken into account in the spectral analysis. GRB 040223 is composed by three peaks, with a soft integrated spectrum well fitted by a power law. The lack of adequate statistics prevents us from testing more complex models or detecting any kind of spectral evolution. Our results are fully in agreement with those reported by Filliatre et al. (2006).

This burst was observed by XMM-Newton $5 \mathrm{~h}$ after the trigger, and a fading afterglow was detected (Tiengo et al. 2004).

\subsection{GRB 040323}

This GRB was single-peaked and quite intense. Nevertheless it is apparently a dark burst, as no afterglow was detected in the following hours and days. A tentative detection of an optical counterpart (Gal-Yam et al. 2004) has not been confirmed. The spectrum of the burst is well described by a CPL model, with a cutoff energy $E_{0}=120_{+84}^{-39} \mathrm{keV}$ (see Table 4). The F-test gives a probability of only $2 \times 10^{-4}$ that the improvement obtained using the CPL model instead of the power law is obtained by chance. We report a contour plot of the CPL fit parameters in Fig. 6.

To our knowledge, there is no other published work about this burst made using ISGRI data. Foley et al. (2008) used SPI data, fitting the spectrum of GRB 040323 with a power law. They found a best-fit photon index of $1.44 \pm 0.18$. They could not detect the cutoff, due to the poor statistics of their spectrum. As a consequence they found a fluence slightly higher than ours.

\subsection{GRB 040403}

This burst is quite faint and soft, with an integrated spectrum well fitted by a power law with a photon index $1.84_{+0.16}^{-0.15}$. Our results agree with those reported in Mereghetti et al. (2005), who
Table 5. Results of fitting some spectra of INTEGRAL GRBs with a quasi-thermal model.

\begin{tabular}{lccccc}
\hline \hline$\#$ & Name & \multicolumn{3}{c}{ Quasi-thermal model } \\
& & Photon index & $\boldsymbol{k} \boldsymbol{T}$ & $\chi_{\text {red }}^{2}$ & d.o.f. \\
& & & {$[\mathrm{keV}]$} & & \\
\hline 2 & 021219 & $1.47_{+0.29}^{-0.38}$ & $50_{+3}^{-2}$ & 1.0 & 44 \\
13 & 040422 & $2.20_{+0.52}^{-0.32}$ & $14_{+3}^{-3}$ & 1.1 & 44 \\
24 & 050502 & $1.89_{+0.43}^{-0.26}$ & $22_{+2}^{-3}$ & 1.0 & 44 \\
28 & 050525 & $2.13_{+0.07}^{-0.06}$ & $16_{+3}^{-3}$ & 1.3 & 31 \\
38 & $060428 \mathrm{C}$ & $1.70_{+0.20}^{-0.15}$ & $19_{+2}^{-3}$ & 0.8 & 44 \\
39 & 060901 & $1.70_{+0.35}^{-0.21}$ & $29_{+5}^{-5}$ & 0.9 & 44 \\
42 & 061025 & $1.50_{+0.54}^{-0.30}$ & $21_{+4}^{-4}$ & 1.1 & 44 \\
43 & 061122 & $1.85_{+0.16}^{-0.11}$ & $19_{+3}^{-3}$ & 0.8 & 44 \\
\hline
\end{tabular}

found a slightly higher fluence, probably due to the non optimal calibration used at that time. We did not detect significant spectral evolution. Unfortunately this GRB happened during a fullMoon night, so optical follow-ups were difficult and no optical afterglow was detected. Nevertheless, a quite deep limit in magnitude was obtained $17 \mathrm{~h}$ after the trigger $(R>24.2$, Mereghetti et al. 2005), indicating a rather faint afterglow, as seen in other soft and faint bursts. No X-ray or radio follow-up observations were performed.

\subsection{GRB 040422}

This GRB was seen $9.4^{\circ}$ off-axis, but it was bright enough to obtain a good quality spectrum that showed evidence of a cutoff at $E_{0}=45_{+25}^{-13} \mathrm{keV}$ (see Table 4). The F-test gives a probability of only $10^{-5}$ that the improvement of the fit with respect to the power law is obtained by chance. Our results are compatible with those reported by Filliatre et al. (2005): they found a similar cutoff value, but they used the Band model fixing the high-energy photon index to -4 . We have also obtained a good fit using the QT model, with parameters fully compatible with those reported by Foley et al. (2008) but with smaller errors. The F-test is not applicable here to compare this fit with that obtained with the power law (Protassov et al. 2002). We obtain the same $\chi_{\text {red }}^{2}$ using both the CPL model and the QT model, but the latter model requires one more parameter.

The afterglow was detected by Filliatre et al. (2005) in the near infrared $2 \mathrm{~h}$ after the burst. They also identified the host galaxy, but it is too faint to measure the redshift.

\subsection{GRB 040624}

This burst was observed $12^{\circ}$ off-axis, so that, despite its intrinsic brightness, it resulted in quite a low signal to noise level. Its spectrum is well described by a power law, with a photon index fully compatible with that reported by Filliatre et al. (2006). These authors derived a duration for the burst of $T_{90}=46 \mathrm{~s}$, while we found $T_{90}=27 \mathrm{~s}$. Moreover they reported a fluence nearly two times higher than ours. We do not understand the reason for these differences, but we think they could be due to an inadequate calibration.

This GRB was promptly observed from the ground, but no optical afterglow was detected down to the magnitude $R>23.8$ only half a day after the trigger, despite the very low Galactic 


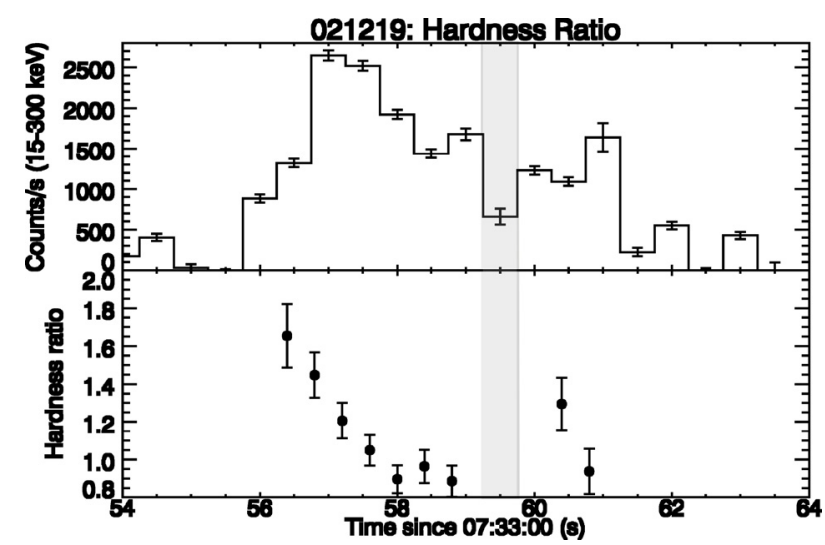

Fig. 7. Spectral evolution of GRB 021219: the hard-to-soft evolution is clearly visible. The hardness ratio is defined as $\mathrm{H} / \mathrm{S}$, where $\mathrm{H}$ is the count rate in the $50-300 \mathrm{keV}$ range and $\mathrm{S}$ is the count rate in the $15-50 \mathrm{keV}$ range. The gray region is affected by a telemetry gap, so no meaningful hardness ratio could be computed.

absorption present in the burst direction. Thus this GRB could be intrinsically dark (Filliatre et al. 2006).

\subsection{GRB 040730}

This GRB was located very close to the Galactic plane, and only $\sim 34^{\circ}$ from the Galactic center direction. In this direction the extinction is very intense, so it is not surprising that no afterglows were detected at any wavelength. The spectrum of this burst is well fitted by a power law, with results in full agreement with those reported by Foley et al. (2008).

\subsection{GRB 040812}

This is the first burst that occurred in the field of view of the JEM-X instrument. It was weak, but quite soft so that it was well detected in the JEM-X energy band (3-35 keV). We have analyzed the JEM-X data finding the burst best position at coordinates RA $=246.463$, Dec $=-44.714$ (J2000), with a $2^{\prime}$ error radius $(90 \%$ c.l.). This position is fully in agreement with the ISGRI one. The spectrum we have extracted from ISGRI data is well described by a power law. Adding the JEM-X data does not change significantly the best fit parameters, owing to the large statistical errors. An extensive multi wavelength campaign was performed to search for an afterglow candidate. Only the Chandra/ACIS X-ray telescope found convincing evidence of a decaying source inside the IBIS error circle (Patel et al. 2004; Campana \& Moretti 2004). Its coordinates, $\mathrm{RA}=246.5093, \mathrm{Dec}=-44.7304$, are $1.6^{\prime}$ away from the IBIS position, and at 2.1' from the JEM-X position. Following this detection, D'Avanzo et al. (2006) found a decaying optical source and a candidate host galaxy. Due to the lack of emission features from the galaxy they could only derive a tentative redshift range of $0.3 \leq z \leq 0.7$.

\subsection{GRB 040827}

This burst was rather faint and observed at an off-axis angle of $12^{\circ}$, with only $\sim 10 \%$ of the ISGRI detector surface exposed to the source flux. Thus the trigger significance was below the threshold for the automatic alert delivery. The burst was confirmed by an interactive analysis and announced to the scientific community about one hour later (Mereghetti et al. 2004a).
Several follow-up observations were carried out, leading to the detection of the afterglow in the X-ray band with XMM-Newton and in the NIR with the VLT. The accurate NIR position $(\mathrm{RA}=229.25558$, Dec $=-16.14142$, de Luca et al. 2005) is only $0.6^{\prime}$ away from the refined ISGRI position.

The analysis of this burst is made impossible by the presence in the IBIS field of view of the very bright and variable source Sco X-1. This low-mass X-ray binary is detected at $14 \sigma$ above the background in the GRB time interval, and is only $7^{\circ}$ away from the IBIS optical axis. The GRB is detected instead only $10 \sigma$ above the background and is $12^{\circ}$ off-axis, so that the field is dominated by the flux of Sco X-1. The spectra of both the GRB and Sco X-1 extracted at the same time, as requested by the nature of the coded-mask telescope, are distorted and cannot be fitted with reasonably simple models. To better understand the problem we have extracted two spectra of Sco X-1 in two intervals before and after the GRB, lasting $\sim 30 \mathrm{~s}$, like the GRB. Due to the short exposure, Sco X-1 is well detected only in energy bins below $70 \mathrm{keV}$, while its contribution is negligible above that energy. Both spectra are well described by the best fit (averaged) model proposed by Di Salvo et al. (2006). The spectrum extracted during the GRB is compatible with these ones only below $50 \mathrm{keV}$, while it shows a lack of counts in the $50-70 \mathrm{keV}$ energy range. The GRB spectrum presents an excess in the same range. We conclude that the software is not able to properly disentangle the contributions of the two sources around $50 \mathrm{keV}$, so that both spectra are distorted. We have tried to reduce the number of energy bins, and even to extract the spectra only around the peak of the GRB (maximizing the signal to noise ratio), but without results.

\subsection{GRB 040903}

This relatively faint and spectrally soft burst came from a direction close to the Galactic center $\left(l=5.2^{\circ}, b=-1.5^{\circ}\right)$. Due to the presence of a faint ROSAT X-ray source in its error region, the possibility that it was due to a type I X-ray burst from an unidentified low mass X-ray binary was considered in the initial reports (Götz et al. 2004a). However, subsequent analysis (Kuulkers et al. 2004), confirmed here, indicated that a type I $\mathrm{X}$-ray burst origin is rather unlikely. We therefore include this event in our sample of GRBs. Indeed this burst is detected only below $100 \mathrm{keV}$ and, although its poorly constrained spectrum is consistent also with a blackbody, there are several factors at variance with a type I X-ray burst origin. The blackbody temperature of $6.7 \pm 1.1 \mathrm{keV}$ is much higher than that typically observed in X-ray bursts and there is no evidence for a spectral softening. The ISGRI spectrum is also well fit with a power law with photon index $2.9_{+0.46}^{-0.39}$. Finally, no persistent emission from the ROSAT source has been detected by INTEGRAL before or after the burst. Thus we conclude that the nature of this burst is that of an X-ray flash.

\subsection{GRB 041015}

This faint GRB was detected $\sim 10^{\circ}$ off-axis, and its significance was below the threshold for the automatic delivery of the alert message. A manual alert was distribuited about $1 \mathrm{~h}$ later (Mereghetti et al. 2004b). Despite the follow up observations carried out by optical and infrared observatories, no afterglow candidates were detected. 


\subsection{GRB 041218}

The spectrum of GRB 041218 is well described by a power law. Foley et al. (2008) obtained peak flux and fluence values very similar to those reported here, but they were unable to fit the spectrum with a power law and obtained only marginally acceptable fits with the CPL and the QT model. This is probably due to the use of old spectral extraction software and calibration responses not optimized at large off-axis angles. In fact this bright burst was at an off-axis angle of $\sim 13^{\circ}$. The light curve of GRB 041218 shows three main peaks, for which we extracted individual spectra: they are well described by power laws, with photon index respectively $\alpha_{1}=1.52 \pm 0.08, \alpha_{2}=1.49 \pm 0.09$ and $\alpha_{3}=1.72 \pm 0.10$. While the first and the second peaks are almost identical, the third one is slightly softer.

An optical afterglow has been detected, and two breaks in the temporal decay have been reported by Torii et al. (2005).

\subsection{GRB $041219 A$}

Thanks to the long duration of this GRB, the IBAS alert was issued when the burst was still on going (Götz et al. 2004b). This allowed robotic telescopes to detect a prompt optical and IR flash (Blake et al. 2005; Vestrand et al. 2005) whose position was consistent with the IBAS one. GRB 041291A is the longest and brightest GRB in the IBIS sample, and could be studied in detail. For example, using SPI data a high degree of polarization of the prompt emission was reported for this burst, but with low significance (McGlynn et al. 2007). The burst is composed of three distinct emission episodes: two close precursor peaks, and then after a quiescent interval the main GRB emission. The spectra of the two precursors are well described by power laws, with a photon index respectively of $1.38 \pm 0.04\left(\chi_{\text {red }}^{2}=1.46\right.$ with 46 d.o.f.) and $2.16 \pm 0.08$ ( $\chi_{\text {red }}^{2}=0.5$ with 36 d.o.f. $)$. The spectral evolution is evident. Also the spectrum of the GRB is well described by a power law, with a photon index of $1.89 \pm 0.01$ $\left(\chi_{\text {red }}^{2}=1.12\right.$ with 46 d.o.f. $)$. In order to study the spectral evolution, we have divided the GRB in two parts: the first one from $240 \mathrm{~s}$ to $356 \mathrm{~s}$ and the second one from $356 \mathrm{~s}$ to the end of the burst (see Fig. 12). The corresponding spectra can be described with power laws, with a photon index of $1.62 \pm 0.02\left(\chi_{\text {red }}^{2}=1.36\right.$ with 46 d.o.f. $)$ and $2.17 \pm 0.02\left(\chi_{\text {red }}^{2}=1.4\right.$ for 46 d.o.f. $)$ respectively. Again the softening is evident. For a more detailed analysis of this peculiar event, and a comparison with the analysis of SPI data, see Götz et al. (2008, in preparation).

\subsection{GRB 050129}

This very faint GRB was below the significance level requested for the automatic delivery of the IBAS alert. Its spectrum is quite soft, so it is revealed only below $120 \mathrm{keV}$. No afterglow candidates have been detected.

\subsection{GRB 050223}

This GRB was discovered by Swift (Mitani et al. 2005). Just by chance INTEGRAL was observing in the direction of this burst, that was thus detected by the IBIS instrument at an off-axis angle of $14^{\circ}$. The burst triggered the IBAS programs, however the alert was not automatically distributed, because the burst occurred during a short time interval (200 s) in which one of the 8 modules composing the ISGRI detector was switched off (this is done autonomously by the on board software handling the noisy pixels).
Under these circumstances there is the possibility that IBAS incorrectly assigns to the GRB the coordinates of one of its ghost images, so automatic alert distributions are disabled. In the offline analysis it was possible to take these effects into account. Our results are fully compatible with those obtained by Page et al. (2005) with Swift data.

Following the Swift /BAT trigger and the Swift/XRT detection (Giommi et al. 2005), several follow-up observations were carried out. An afterglow was detected with XMM-Newton in the X-ray band (de Luca \& Campana 2005), but not at opti$\mathrm{cal} / \mathrm{NIR}$ wavelengths. A galaxy at redshift $z=0.584$ is contained in the 1.5 arcsec radius error region provided by XMM-Newton (Pellizza et al. 2006). Assuming that this is the GRB host galaxy, the isotropic energy output can be estimated as $10^{51} \mathrm{erg}$, a quite low value. However, the probability of a chance superposition between the putative host galaxy and the X-ray error circle is 19\% (Cobb \& Bailyn 2008).

\subsection{GRB 050502}

This burst was in the fully coded field of view of IBIS, but it occurred just after a satellite slew. This is the reason why time bins before 02:13:56 UT are lacking in the light curve. Moreover there was a telemetry saturation causing data loss at 02:14:10 UT. Thus our measures of $t_{90}$ and fluence are only lower limits. The spectrum has a very good signal-to-noise ratio and is poorly described by a power law $\left(\chi_{\text {red }}^{2}=1.6\right.$ with 46 d.o.f.). A CPL model gives a better fit $\left(\chi_{\text {red }}^{2}=1.0\right.$ with 45 d.o.f.). The F-test gives a chance probability of observing such a $\chi^{2}$ improvement of only $3 \times 10^{-6}$. The measured cutoff energy is $E_{0}=91_{+47}^{-25} \mathrm{keV}$ and the CPL photon index is $\alpha=0.86_{+0.22}^{-0.24}$. These values correspond to a peak energy $E_{\mathrm{p}}=$ $104_{+55}^{-38} \mathrm{keV}$. Our measure is fully compatible with that reported by Schaefer (2007). In Fig. 6 we report the confidence contour for the two parameters of the CPL model. The spectrum of this GRB can also be well described with a QT model $\left(\chi_{\text {red }}^{2}=1.0\right.$ with 44 d.o.f.), with the parameters reported in Table 5.

Only $\sim 23$ s after the IBAS trigger, the ROTSE-IIIb robotic telescope started to observe the field of GRB 050502 and discovered its optical afterglow. Follow-up observations allowed investigators to detect a break in the light curve that seemed to be achromatic (Yost et al. 2006; Guidorzi et al. 2005). Moreover, the Keck-I optical telescope acquired a high-resolution spectrum of the afterglow only $3 \mathrm{~h}$ after the burst, measuring a redshift of $z=3.793$ (Prochaska et al. 2005). This is the first INTEGRAL GRB with both a measured cutoff and a known redshift. With this information we can derive the GRB parameters in the cosmological rest frame, using the procedure described by Amati et al. (2002). Using the total duration of the burst (20 s), we computed the total emitted energy (in the isotropic hypothesis) $E_{\text {iso }}=8.8 \times 10^{52} \mathrm{erg}$. The rest-frame peak energy resulted in $E_{\mathrm{p}}^{\text {iso }}=508_{+289}^{-135} \mathrm{keV}$, while the isotropic (peak) luminosity resulted in $L_{\text {iso }}=2.38 \times 10^{52} \mathrm{erg} \mathrm{s}^{-1}$. We used the standard values for the cosmological parameters $\left(H_{0}=70 \mathrm{~km} \mathrm{~s}^{-1} \mathrm{Mpc}, \Omega_{\lambda}=0.7\right.$ and $\Omega_{\mathrm{m}}=0.3$ ). In Fig. 8 we report our measured values for $E_{\mathrm{p}}$, $E_{\text {iso }}$ and $L_{\text {iso }}$ : we can see that this GRB lies quite well on the Amati and Yonetoky correlations. We note, however, that our value for $E_{\text {iso }}$ could be affected by the unknown duration of the $\mathrm{GRB}$, as mentioned above. 

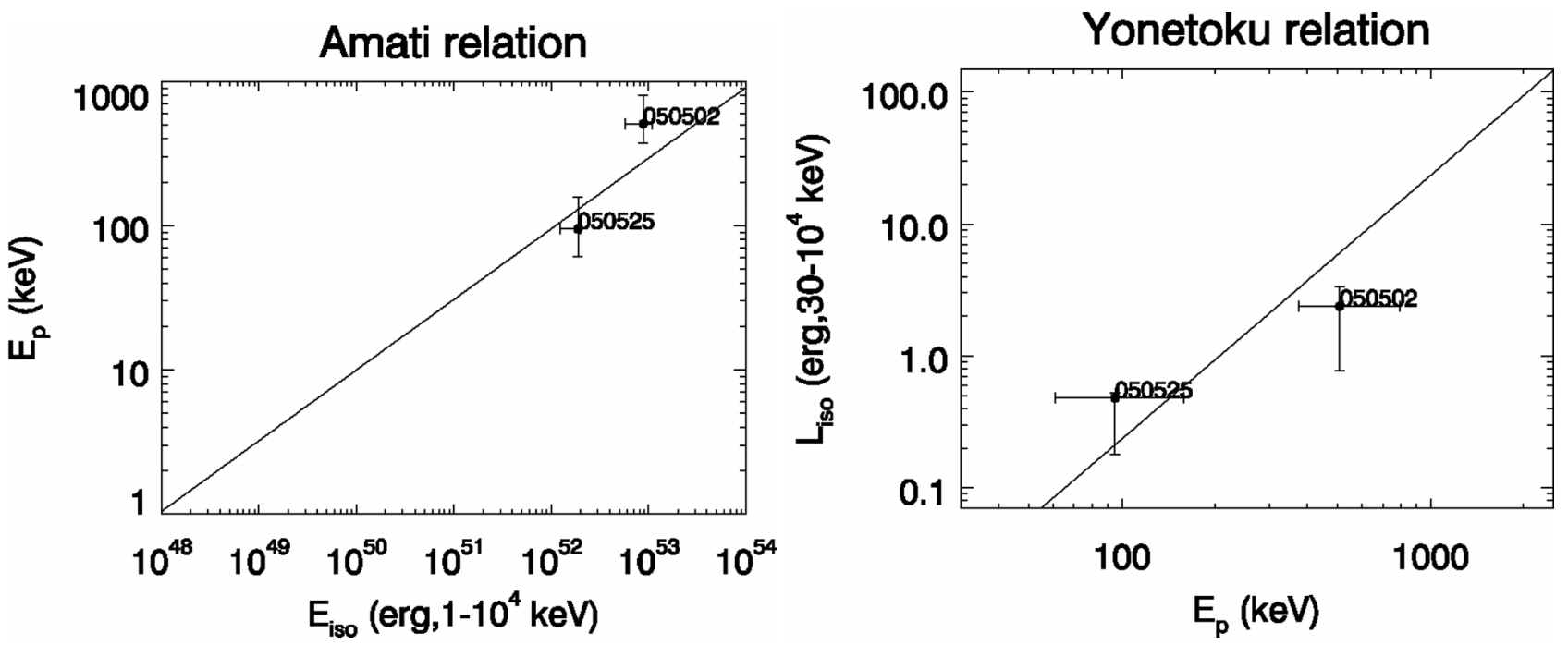

Fig. 8. Position of two INTEGRAL GRBs on the $E_{\mathrm{p}} \mathrm{Vs} E_{\mathrm{iso}}$ (left panel, Amati 2006) and $L_{\text {iso }}$ Vs $E_{\mathrm{p}}$ (right panel, Yonetoku et al. 2004) relations.

\subsection{GRB 050504}

This burst was detected in the fully coded field of view, with the whole detector area exposed to the flux of the source. The resulting good statistics allowed us to measure a spectral cutoff. In fact the CPL model gave a better fit than a power law, with an F-test chance probability of $6 \times 10^{-4}$. We also tried the QT model, but the best fit value for the blackbody normalization is compatible with zero and without any improvement in the fit quality.

Despite the location of this burst at high Galactic latitude $\left(b=+75^{\circ}\right)$, the presence of a 10th magnitude K type star prevented the observation of part of the error region ( $90^{\prime \prime}$ radius). The precise position derived for the X-ray afterglow, later discovered with Swift (Kennea et al. 2005c), is inconsistent with that of the bright star. The upper limits for the optical afterglow are thus rather constraining: independent groups reported values of $R \sim 19$ and $R \sim 21$ a few minutes after the burst (see for example Cenko \& Fox 2005).

\subsection{GRB 050520}

The integrated spectrum of this multi-peaked GRB is well described by a power law. In order to investigate the spectral evolution of the burst, we have divided the emission in three main episodes: from $T_{0}+55 \mathrm{~s}$ to $T_{0}+85 \mathrm{~s}$, from $T_{0}+85 \mathrm{~s}$ to $T_{0}+100 \mathrm{~s}$, and $T_{0}+100 \mathrm{~s}$ to $T_{0}+120 \mathrm{~s}$ (see Fig. 12). The three corresponding spectra are well fitted by power laws, with photon index $1.52 \pm 0.09\left(\chi_{\text {red }}^{2}=1.13\right.$ with 33 d.o.f. $), 1.58 \pm 0.14\left(\chi_{\text {red }}^{2}=1.04\right.$ with 33 d.o.f.) and $1.72 \pm 0.1\left(\chi_{\text {red }}^{2}=1.15\right.$ with 33 d.o.f. $)$, respectively. Thus, there is no evidence of spectral evolution. Spectral differences could be present in the several peaks contained in each of these intervals, but the burst is not bright enough to allow a spectral analysis for each peak.

Swift/XRT observed the field of GRB 050520 about $2 \mathrm{~h}$ after the trigger, detecting a fading X-ray counterpart (Kennea et al. 2005b).

\subsection{GRB 050522}

This is one of the faintest bursts detected with INTEGRAL. No emission above $\sim 40 \mathrm{keV}$ was detected with IBIS/ISGRI for this burst that can thus be classified as an X-ray flash. Swift reported an X-ray source outside the IBAS error region, but subsequent observations showed it to be unrelated to the burst. The same observations led to the discovery of a fading X-ray source inside the error box, tentatively identified with the afterglow of this burst (Capalbi et al. 2005). However, the variability of this source has not been confirmed, so the identification remains uncertain.

\subsection{GRB 050525A}

This very bright burst, discovered by Swift, occurred by chance within the IBIS field of view, although at a very large off-axis angle $\left(14.7^{\circ}\right)$. A telemetry saturation caused a gap in its light curve. The spectrum of this burst cannot be well fitted by a power law, and requires a CPL or a QT model. Our best-fit parameters of the CPL model are compatible with those obtained with Swift (Cummings et al. 2005) and with Konus-WIND (Golenetskii et al. 2005). The fit with the QT model is good, but gave a slightly worse $\chi^{2}$ with one degree of freedom less than the CPL model.

The high level of coding noise in the deconvolved image, due to the very large off-axis position of the burst, and the simultaneous presence of Cyg X-1 in the field of view, conspired to make GRB 050525A undetectable in real time by the IBAS programs. This was unfortunate, since the Swift localization had a delay of six minutes and the initial part of the optical afterglow was missed by ground based telescopes. The ROTSE-III and TAROT robot telescopes detected the optical afterglow at $R \sim 15$ ten minutes after the burst (Rykoff et al. 2005; Klotz et al. 2005), while earlier optical data were obtained with UVOT on board Swift (Holland et al. 2005). Further observations led to the determination of the redshift $z=0.6$ (Foley et al. 2005) and to the detection of the radio afterglow (Cameron \& Frail 2005). The rest frame cutoff energy is $E_{0}^{\text {rest }}=133_{+66}^{-36} \mathrm{keV}$, the isotropic emitted energy of $E_{\text {iso }}=2.1 \times 10^{52}$ erg and the peak luminosity $L_{\text {iso }}=3.4 \times 10^{52} \mathrm{erg} \mathrm{s}^{-1}$. All these values are compatible with those found with Swift (Nava et al. 2006). In Fig. 8 we report our measured values for $E_{\mathrm{p}}, E_{\text {iso }}$ and $L_{\text {iso }}$ : we can see that GRB 050525 lies on both the Amati and the Yonetoku correlations. 


\subsection{GRB 050626}

This GRB was nearly on axis, lying also within the field of view of the OMC optical telescope. IBAS correctly localized the burst in the IBIS image and a telecommand to place a CCD window at the burst coordinates was automatically sent to the OMC. Unfortunately, the GRB was at only 2' from one of the brightest stars in the sky, $\alpha$ Crucis, that has a magnitude of 0.8 . Thus the region where the GRB took place is severely affected by saturation by this very bright star, making the OMC data for this burst useless. This burst was also detected by JEM-X at coordinates $\mathrm{RA}=186.74$, Dec $=-63.13,25$ arcsec away from the ISGRI position. The ISGRI spectrum is well fitted by a power law. Adding the JEM-X spectrum does not change significantly the best fit parameter. No afterglow has been detected (Gorosabel et al. 2005; Mangano et al. 2005).

\subsection{GRB 050714}

The spectrum of this faint burst was well fitted by a power law. The light curve is composed of three peaks, but the burst is too weak to perform a time-resolved spectral analysis. A few minutes after the onset of the burst two sources were proposed as optical counterparts (Klose et al. 2005a), but these detections were not confirmed. About $14 \mathrm{~h}$ later an X-ray candidate was detected using Swift/XRT (Racusin et al. 2005), and soon after an optical source was discovered in the XRT error circle (Klose et al. 2005b). The optical variability of the source has not been confirmed, so its identification with the afterglow has to be taken with care.

\subsection{GRB 050918}

This burst was at $14^{\circ}$ off-axis, with only a few percent of the detector surface exposed. No prompt IBAS Alert had been issued for this burst because its significance was below the threshold for automatic alert delivery. The light curve shows two distinct emission episodes, separated by a quiescent period of $\sim 180 \mathrm{~s}$. The missing data visible in the light curve are due to telemetry saturation. We have extracted the spectrum excluding the quiescent period. It is well fitted by a power law. Contrary to the results of Foley et al. (2008), we could not find improvements in the fit using the CPL or QT models. This difference is probably due to the better calibration for large off-axis angles now available. We have also extracted the spectra of the two emission episodes separately. Although they are separated by a long quiescent period, they are both fitted with practically the same power law photon index: $\alpha=1.72 \pm 0.14$ for the first episode $\left(\chi_{\text {red }}^{2}=1.04\right.$ with 30 d.o.f.), and $\alpha=1.76 \pm 0.12$ for the second episode ( $\chi_{\text {red }}^{2}=0.6$ with 30 d.o.f.).

A follow-up observation of this burst was carried out with Swift, revealing a previously uncatalogued faint X-ray source inside the 2.8 arcmin radius INTEGRAL error circle (Kennea et al. 2005a). There was no evidence of fading in this source, however as this observation occurred more than 2 days after the burst, this result is expected.

\subsection{GRB 050922}

The significance of this faint burst was below the threshold for automatic alert delivery. The burst is detected only up to $100 \mathrm{keV}$, with a very low signal-to-noise ratio, so its spectrum is affected by large uncertainties. No afterglow was detected (Sonoda et al. 2005).

\subsection{GRB 051105B}

This faint burst was observed only $2.5^{\circ}$ off-axis. This position is within the field of view of JEM-X, but just outside that of the OMC. The position derived with JEM-X, RA $=9.442, \mathrm{Dec}=$ -40.495 , is 1.5 arcmin away from the ISGRI position. Due to the low significance of the detection at soft X-ray energies, is not possible to extract a spectrum with JEM-X data. The ISGRI spectrum is well fitted by a power law.

Despite the position of the burst, it was observed with optical instruments only a few hundred seconds after its onset, no afterglow was detected (Kinugasa \& Torii 2005; Torii \& Mereghetti 2005; Distefano et al. 2005). The field was observed two days later with XRT and UVOT onboard Swift. Again, no afterglow was detected (Mineo et al. 2005; Blustin et al. 2005).

\subsection{GRB $051211 B$}

This GRB was observed about $10^{\circ}$ off-axis. Its spectrum is well described by a power law. This burst was promptly observed by optical telescopes and by Swift/XRT. An afterglow candidate was detected in X-rays (La Parola et al. 2005), then confirmed in the optical (Jelinek et al. 2005). There has been also a detection at radio wavelength, but it was not confirmed (Frail 2005).

\subsection{GRB 060114}

This faint GRB was only $2.6^{\circ}$ from the IBIS optical axis. Thus it was in the JEM-X field of view, but too faint to be detected. Its spectrum is well fitted by a simple power law, with a quite hard photon index. No afterglow was detected.

\subsection{GRB 060130}

This is another faint GRB, seen near the IBIS optical axis $\left(3^{\circ}\right.$ off-axis). Despite it being inside the JEM-X field of view, it is not detected due to its faintness. Its spectrum is well fitted with a power law. No afterglow was detected.

\subsection{GRB 060204}

This faint GRB was observed at the edge of the fully coded field of view of IBIS, and slightly outside of the field of view of JEM-X. Its spectrum is well described by a power law. No afterglow was detected.

\subsection{GRB $060428 C$}

This bright GRB occurred during a short period in which IBAS was not running. It was detected off-line by Grebenev \& Chelovekov (2007). The light curve shows several peaks, with no (or very short) periods of quiescence. The gaps visible after the highest peak is due to telemetry saturation. The integrated spectrum cannot be described by a power law $\left(\chi_{\text {red }}^{2}=1.9\right.$ with 46 d.o.f.), while is well fitted by a CPL model with photon index $\alpha=0.86 \pm 0.2$ and cutoff energy $E_{0}=85_{+34}^{-19} \mathrm{keV}$ $\left(\chi_{\text {red }}^{2}=0.9\right.$ with 45 d.o.f.). The F-test chance probability of obtaining such an improvement is only $2 \times 10^{-8}$. The spectrum can be described also by a QT model with blackbody temperature $k T=19.47_{+2.34}^{-2.56} \mathrm{keV}$ and photon index $\alpha=1.70_{+0.20}^{-0.15}$. We have also perfomed a time-resolved spectral analysis, dividing the burst in three parts, and fitting with a power law, a CPL and a QT model. The results, reported in Table 1, are fully in 
060901: Confidence contours

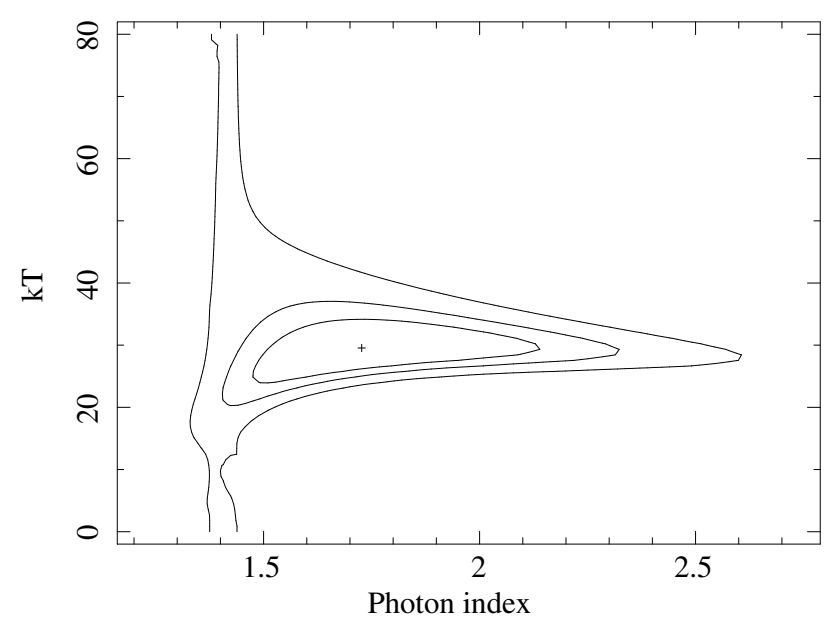

Fig. 9. Contour plot of the QT model parameters applied to GRB 060901. The contours are at $68 \%, 90 \%$ and $99 \%$ confidence levels for two parameters of interest.

agreement with those of Grebenev \& Chelovekov (2007). Note, however, that those authors gave errors at a $1 \sigma$ level. The GRB shows a clear spectral evolution, going from quite a soft spectrum to a harder one, then coming back to soft emission.

Owing to the lack of notification by the IBAS system no follow-up observations were done.

\subsection{GRB 060901}

This burst was observed $14^{\circ}$ off-axis, with only a few percent of the detector surface exposed to the flux from the source. In the light curve there is a gap due to telemetry saturation. The spectrum is well described by a power law ( $\chi_{\text {red }}^{2}=1.1$ with 46 d.o.f.). While the CPL model does not improve the fit, the QT model describes the spectrum slightly better $\left(\chi_{\text {red }}^{2}=0.9\right.$ with 44 d.o.f. $)$ than the power law. However, the contour plot in Fig. 9 shows that the best fit parameters are not well constrained. The limited fit improvement and the poor constraints of the parameters indicate that probably this result is not significant. Foley et al. (2008) did not succeed in fitting the spectrum of GRB 060901 with a power law, and use a QT model with parameters compatible with ours. However, they obtain larger uncertainties and a worse $\chi_{\text {red }}^{2}$. This again could be due to the different calibration they used, that is not optimal in analyzing bursts with such large off-axis angles. GRB 060901 was also detected by Konus-Wind (Golenetskii et al. 2006a). They found that its integrated spectrum is well fitted by a Band model in the range $20 \mathrm{keV}-2 \mathrm{MeV}$, with $\alpha=0.77_{+0.23}^{-0.26}, \beta=2.31_{+0.36}^{-0.18}$ and $E_{0}=156_{+46}^{-71} \mathrm{keV}$.

An afterglow candidate has been discovered by Swift/XRT (Racusin et al. 2006). A tentative optical counterpart has been also proposed (Wiersema et al. 2007), but not confirmed.

\subsection{GRB 060912B}

This is a very long and faint GRB, with a slow rise and and a slow decay. Its spectrum is well fitted by a power law, while there are not enough statistics to constrain both the CPL and the QT models. No afterglow was detected.

\subsection{GRB 060930}

This is another quite faint GRB, detected $8.5^{\circ}$ off-axis. Its spectrum is well described by a power law, with no improvement in the fit using the CPL model or the QT model. No afterglow was detected.

\subsection{GRB 061025}

This burst was detected $8.4^{\circ}$ away from the IBIS optical axis. Its spectrum is not described very well by a power law $\left(\chi_{\text {red }}^{2}=\right.$ 1.6 with 46 d.o.f.), while a CPL model describes it much better $\left(\chi_{\text {red }}^{2}=1.1\right.$ with 45 d.o.f., F-test chance probability of $\left.1 \times 10^{-5}\right)$. We can obtain a good fit with the QT model too, but with one more parameter $\left(\chi_{\text {red }}^{2}=1.1\right.$ with 44 d.o.f.). We obtain best fit parameters for the QT model compatible with that of Foley et al. (2008), but with smaller errors. On the contrary, they could not fit the spectrum with a CPL model, probably because of their software version: OSA 5.1 is not optimal in extracting spectra from a source with an off-axis angle so large.

Following the localization provided by the IBAS system, the Swift/XRT detected the X-ray counterpart (Mineo et al. 2006) and the ROTSE III optical telescope confirmed it (Yuan \& Rykoff 2006).

\subsection{GRB 061122}

This is a bright GRB, observed at $8.2^{\circ}$ from the IBIS optical axis. This burst also triggered Konus-Wind (Golenetskii et al. 2006b). Two gaps due to telemetry saturation are visible in the light curve, so that the peak flux reported in Table 2 should be regarded as a lower limit. The spectrum is poorly described by a power law model $\left(\chi_{\text {red }}^{2}=1.6\right.$ with 39 d.o.f.), while we obtained a good fit with the CPL model ( $\chi_{\text {red }}^{2}=0.8$ with 45 d.o.f.). The F-test probability of obtaining a similar improvement by chance is $2 \times 10^{-7}$. We measured a photon index $\alpha=1.24 \pm 0.16$ and a cutoff energy $E_{0}=122_{+60}^{-31} \mathrm{keV}$. Also the spectrum measured by Konus-Wind is well fitted by a CPL model, with $\alpha=1.03_{+0.06}^{-0.07}$ and $E_{0}=160_{+8}^{-7} \mathrm{keV}$ (Golenetskii et al. 2006b). These values are compatible with ours. Our spectrum also could be fitted with the QT model, with a similar quality $\left(\chi_{\text {red }}^{2}=0.8\right.$ with 43 d.o.f.). Foley et al. (2008) obtain the best description of the spectrum of GRB 061122 using a QT model, while they were not able to constrain the cutoff energy in the CPL model. Moreover, their best fit value for the blackbody temperature in the QT model $(12 \pm 3 \mathrm{keV})$ is only marginally compatible with ours $(19 \pm 3 \mathrm{keV})$. This could be due again to the off-axis angle of this burst.

An afterglow has been detected at X-ray wavelengths (Halpern 2006) and in the optical band (Halpern \& Armstrong 2006).

\subsection{GRB 070903}

This long and faint GRB was observed with an off-axis angle of $7.6^{\circ}$. Its integrated spectrum is affected by large statistical errors due to the low signal to noise ratio. It is well described by a power law $\left(\chi_{\text {red }}^{2}=1.3\right.$ with 16 d.o.f.), while neither the CPL model nor the QT model give a better fit.

The X-ray afterglow of this GRB has been observed by Swift/XRT (Vergani et al. 2007). 


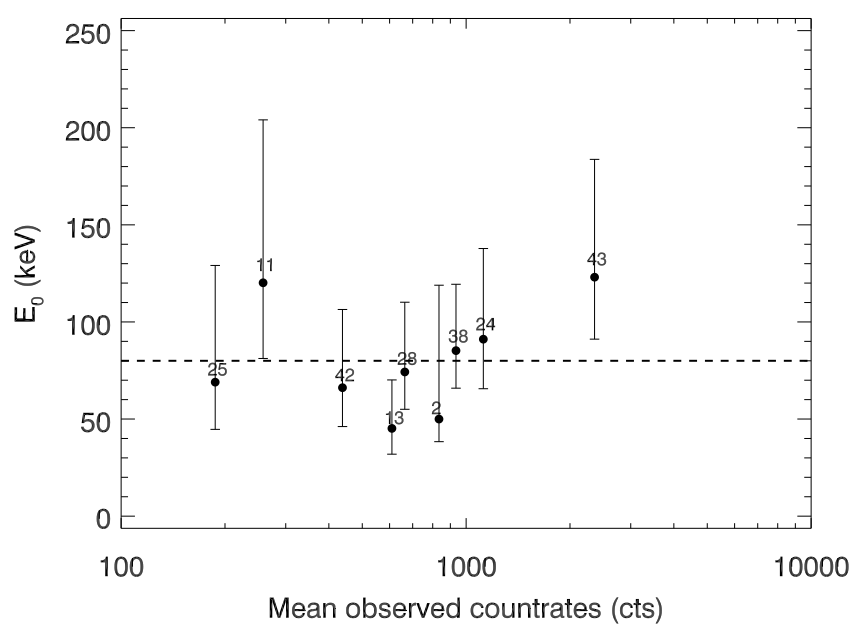

Fig. 10. Cut-off energy $E_{0}$ as a function of the mean countrate (background subtracted, not renormalized) measured during each GRB. The error bars are at the $90 \%$ confidence level for a single parameter of interest. The dashed line is the mean cutoff energy $\left\langle E_{0}\right\rangle=80 \mathrm{keV}$.

\subsection{GRB 070311}

This burst was observed at a position $12^{\circ}$ away from the IBIS optical axis. Its spectrum is well described by a power law, with no improvement adopting other models. Our results are compatible with that of Sazonov et al. (2007) and Guidorzi et al. (2007b).

Thanks to the prompt localization provided by the IBAS system, the REM telescope detected the afterglow of GRB 070311 only $51 \mathrm{~s}$ after the onset of the GRB (Covino et al. 2007). The afterglow was later observed by other observatories at optical wavelengths, and by the Swift/XRT at soft X-ray energies (Guidorzi et al. 2007a). The light curve of the afterglow showed a major rebrightnening and other interesting features, described in detail in Guidorzi et al. (2007b).

\section{Discussion}

\subsection{Spectral results}

We have measured the cutoff energy $\mathrm{E}_{0}$ in 9 out of 43 GRBs, corresponding to $\sim 20 \%$ of our sample. The values of $\mathrm{E}_{0}$ are in the range $50-150 \mathrm{keV}$, and are clustered around $80 \mathrm{keV}$ (see Fig. 10). This is not surprising, because most of the spectra have a significant signal from $18 \mathrm{keV}$ to $300 \mathrm{keV}$, but the spectral bins above $200 \mathrm{keV}$ usually have large statistical errors. In order to constrain the cutoff energy, a significant number of energy bins with good statistics are needed both below and above the cutoff value. Thus, the limited bandpass of ISGRI introduces a bias in the cutoff measurement, as also seen in similar instruments, like Swift/BAT (see, i.e., Ghirlanda et al. 2008). Sakamoto et al. (2008) studied a large sample of BAT bursts detecting a cutoff energy in 32 long GRBs out of 216 , a percentage $(\sim 15 \%)$ even smaller than that found in our sample.

The largest GRB sample with good spectral information currently available is that obtained with the BATSE/CGRO experiment. It demonstrates that the majority of GRBs are well fitted by the Band model (Band et al. 1993; Preece et al. 2000). Analyzing the subsample of very bright GRBs, with peak flux $>10 \mathrm{ph} \mathrm{cm}^{-2} \mathrm{~s}^{-1}$ and fluence $>10^{-5} \mathrm{erg} \mathrm{cm}^{-2}(20-2000 \mathrm{keV})$, Kaneko et al. (2006, hereafter K06) found that the average values for the low- and high-energy photon index are $\alpha \simeq 1.1$ and $\beta \simeq 2.3$, respectively. The two photon index distributions are clustered around these values and are well separated. Our photon index distribution (Fig. 5) peaks around 1.6, a value between the average values of $\alpha$ and $\beta$. A similar result was found for Swift/BAT GRBs (Sakamoto et al. 2008). If the results found by K06 using only the brightest bursts apply to the whole GRB population, we can conclude that we are unable to see some cutoff energies either because they are within the ISGRI energy range but the spectra do not have high enough statistics, or because the cut-off lies outside the ISGRI energy band. In both cases the observed spectrum would have some curvature, so that in both cases the best fit with a single power law would give a photon index intermediate between $\alpha$ and $\beta$.

To estimate the number of undetected cutoffs in our ISGRI spectra, we should know the real distribution of the cutoff values. From the existence of correlations between the energetics of a burst and its spectral parameters (for example the Amati relations, Amati et al. 2002), we know that the distribution of $E_{0}$ is not independent of the fluence distribution of the sample. Only four GRBs of our sample satisfy the brightness criteria of K06, so the cutoff energy distribution derived by those authors is not useful for our purposes. Nava et al. (2008) extended the spectral analysis of BATSE bursts to lower fluences, down to $F \geq 10^{-6} \mathrm{erg} \mathrm{cm}^{-2}(20-2000 \mathrm{keV})$, and found that $35 \%$ of them have a cutoff below $150 \mathrm{keV}$. In our sample there are 30 bursts above this fluence ${ }^{2}$, and the 9 bursts for which we could measure the cutoff are in this sub-sample (corresponding to the $30 \%$ ). The photon index distribution of the other 21 bursts of this subsample is again centered on $\alpha \sim 1.6$. Thus we conclude that we are probably not missing more than a few cutoffs in the subsample with $F \geq F_{0}$, so we have not measured a cutoff energy for most of the remaining 21 bursts because it lies above $150 \mathrm{keV}$ or below $20 \mathrm{keV}$. We can also say that the population of bursts observed by ISGRI with $F \geq F_{0}$ has spectral properties compatible with those observed by BATSE. For the burst with a fluence lower than $F_{0}$ we cannot say if we are missing the measurement or if the cutoff is outside our band.

\subsection{Spectral evolution}

When the fluence of the burst was high enough, we performed a time-resolved spectral analysis that showed different kinds of spectral evolution. In particular, we can divide the spectrally evolving bursts in two classes: single-peaked bursts with spectral evolution, and multi-peaked bursts with spectral differences between the peaks. In the first class we detected only one case of the classical hard-to-soft evolution (GRB 021219). In the second class we detected hard-to-soft evolution (GRB 041218, GRB 041219), soft-to-hard evolution (GRB 040106), more complex evolution (GRB 030320, GRB 060428C) and also one case of no evolution at all (GRB 050918). All these cases are described individually in Sect. 3.

\subsection{Peak fluxes distribution}

In Fig. 11 we show the normalized integral distributions of fluences and peak fluxes for both ISGRI and BAT. The two instruments are very similar from several points of view, with the most important difference being the larger field of view covered by BAT. Thus we expect that the burst populations seen by the two instruments should be very similar. As discussed above, this

\footnotetext{
${ }^{2}$ For a typical spectrum with $\alpha=1.1, \beta=2.3$, and $E_{0}=200 \mathrm{keV}$, the fluence threshold used by Nava et al. (2008) translates to $F \geq F_{0}=$ $4.5 \times 10^{-7} \mathrm{erg} \mathrm{cm}^{-2}$ in our energy band $(20-200 \mathrm{keV})$.
} 

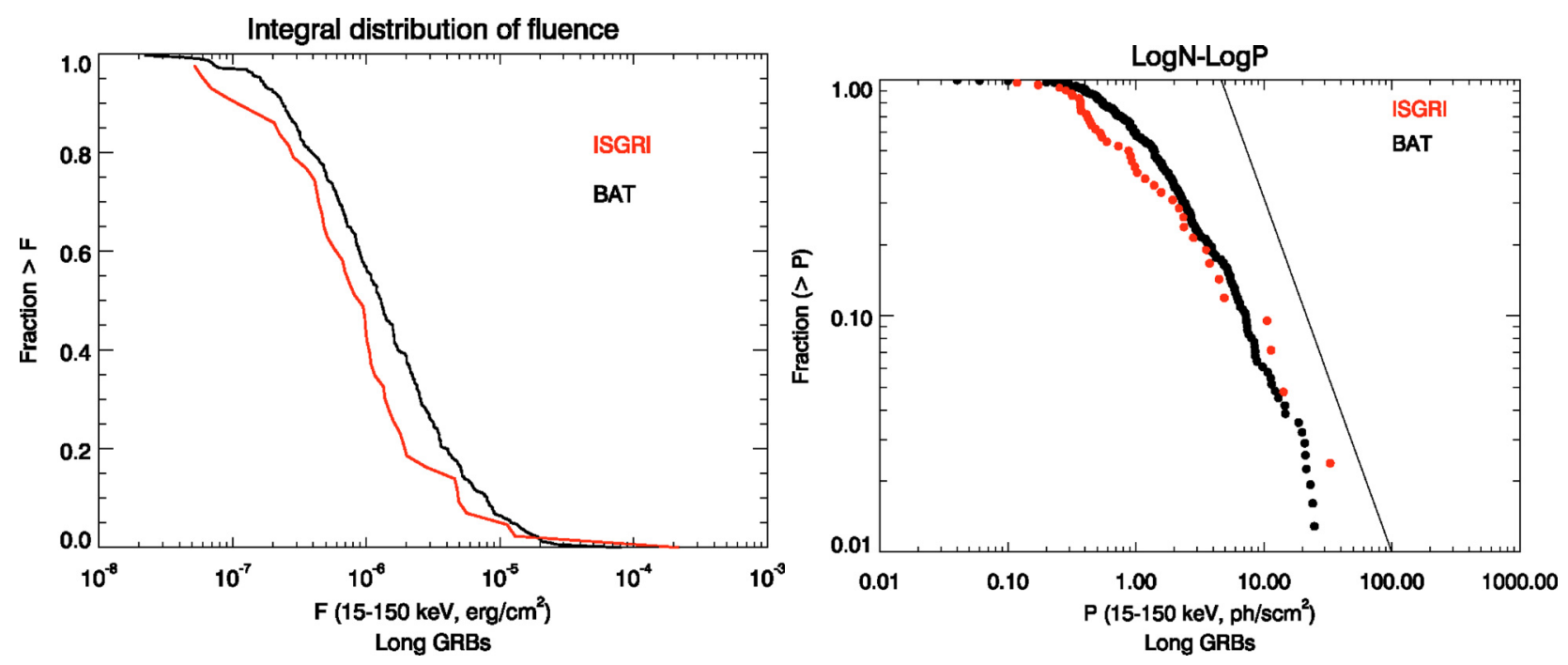

Fig. 11. Normalized integral distributions of fluences (left) and peak fluxes (right) for both ISGRI and BAT.

seems to be true from the spectral point of view. To check if the distributions of fluence and peak flux of the two samples also are similar, we applied a Kolmogorov-Smirnoff (KS) test to the data of Fig. 11. This gave a probability of $12 \%$ that the ISGRI and BAT fluences are drawn from the same distribution, while for the peak fluxes the probability is $3 \%$. The latter result suggests that the two populations are somewhat different, with ISGRI detecting fainter GRBs than BAT. However, larger samples are required to eventually confirm this possible difference.

\subsection{The rate of short bursts}

The IBAS system detected 2 out of 56 short hard bursts, corresponding to $\sim 3.5 \%$, while Swift/BAT found $\sim 8 \%$. This small difference is consistent with the small statistics of the samples. However, the BATSE experiment onboard CGRO found that 1 burst out of 4 was a short hard GRB (25\%). This discrepancy is more compelling. We have to take into account, however, that BATSE was better suited to detect hard bursts: it was based on a different technology than both ISGRI and BAT, and it had a broader bandpass and a larger effective area at high energy. This fact alone could explain the difference in the measured rate of short hard bursts. We will soon have an independent measurement of this rate by the Gamma-ray Burst Monitor (GBM) onboard Fermi.

\subsection{On the relation between spectral lags and peak fluxes}

Peaks of GRBs occur at slightly different times in different energy bands (Cheng et al. 1995). In general they migrate to later times and become wider going to lower energy, although some GRBs with the opposite trend have been observed (Chen et al. 2005). This so-called "spectral lag" correlates with the peak flux of the GRBs (Norris et al. 2000; Norris 2002; Tsutsui et al. 2008): long spectral lags have been measured mainly in GRBs with low peak fluxes, while short spectral lags have been observed in bursts with both high and low peak fluxes. Based on these findings, one can divide GRBs in three groups: bright with short lags, faint with short lags, and faint with long lags.

Foley et al. (2008) measured the spectral lags of INTEGRAL GRBs, and divided the sample in two classes: long-lag GRBs $(\Delta t \gtrsim 0.75 \mathrm{~s})$ and short-lag GRBs $(\Delta t \lesssim 0.75 \mathrm{~s})$. Based on peak fluxes taken from literature or, when unavailable, analyzing the data themselves, they could verify that the three groups mentioned above are also visible in the INTEGRAL GRB sample. In some cases we found GRB peak fluxes quite different from those used by these authors. We therefore repeated their analysis, using their time-lag values, but the peak fluxes computed by us in a consistent way for all the GRBs. We found that the conclusions on the spectral lag-peak flux correlation in INTEGRAL GRBs found by Foley et al. (2008) are confirmed.

\subsection{Spatial distribution}

While the whole INTEGRAL GRB sample is isotropically distributed, the sub-sample of bursts with long lag and low luminosity is spatially correlated with the supergalactic plane (Foley et al. 2008). This result, which supports the existence of a local GRB population, relies on an accurate estimate of the INTEGRAL sky coverage. In computing the sky coverage, these authors used the INTEGRAL exposure maps, but neglected other factors affecting the sensitivity of GRB triggers, thus potentially biasing the observed sky distribution of the faintest bursts (which are indeed those for which an anisotropy was found).

Therefore, to better investigate this interesting point, we computed an IBIS exposure map by also taking into account the sensitivity variations of the IBAS triggers. These are dominated by changes in the background level resulting from the presence of bright sources in the field of view, particle induced events such as solar flares or radiation belt passages, as well as the long term background increase during the mission due to the solar cycle. Based on the actual ISGRI background level measured in each pointing, we rescaled the exposure time with respect to that of an ideal reference pointing (i.e. a high galactic latitude pointing performed at the beginning of the mission and without strong sources in the field of view). The effective exposure time, $t_{\mathrm{eff}}$, has been computed as $t_{\mathrm{eff}}=t_{0} \cdot\left(\sqrt{b k g_{i}} / \sqrt{b k g_{\mathrm{ref}}}\right)$, where $b k g_{i}$ is the background level for the considered pointing, $b k g_{\text {ref }}$ is the reference background, and $t_{0}$ is the raw exposure time. As an example, the effective exposure time with this improved calculation gives $\sim 12 \mathrm{Ms}$ for the Galactic Center region, compared to $\sim 17$ Ms obtained with the simple time exposure map.

After having computed the improved exposure map, we simulated a population of 50000 GRBs uniformly distributed over 
the entire sky following our new map. For this burst population we derived the quadrupole moment with respect to the supergalactic plane, in order to derive our bias, which is $Q=0.046$. Then we derived the GRB quadrupole moment for the 11 longlag GRBs which results in $Q=-0.225 \pm 0.089$, while for all INTEGRAL GRBs we obtain $Q=0.039 \pm 0.042$. Once one subtracts the bias, one obtains $Q=-0.271 \pm 0.089$ for long lag GRBs and $Q=-0.007 \pm 0.042$ for the whole sample. These results confirm the findings of Foley et al. (2008), but with our improved method we enhanced the statistical significance of the quadrupole moment for long lag bursts from $\sim 2.5$ to $\sim 3 \sigma$.

\section{Conclusions}

We have provided a complete spectral catalogue of the publicly available INTEGRAL GRBs, based on IBIS data. By using the latest available software and calibration, we were able to derive accurate spectral parameters for all the bursts in a coherent way, superseding and harmonising all the previous results that could be found in literature. In addition, the spectral evolution of the bursts could be studied in detail for most of the sources.

By computing an accurate exposure map, which takes into account the variation of the instrumental background over our dataset, we were able to confirm, with a higher significance, the clustering of faint, long-lag bursts around the supergalactic plane, supporting the existence of a local GRB population.

We have shown that, despite the good ISGRI sensitivity, which is slightly better then the BAT one, only for a limited number of bursts could the spectral description go beyond a simple power law. Indeed, our analysis and other works in the literature have shown that, despite the increasing number of GRBs with measured redshift, provided mainly by Swift, the number of bursts for which both good spectral and redshift information are available is still limited. Although IBIS has a slightly broader band pass, and better chances to determine $E_{\text {peak }}$ with respect to BAT, it is limited by a smaller field of view, and by its pointing strategy biased towards the low Galactic latitudes, where optical absorption is severe. This explains the low redshift determination rate for INTEGRAL GRBs $(\sim 5 \%)$.

The INTEGRAL and Swift experience has shown that wide field coded mask instruments are a powerful tool for GRB detection and localization, but the lack of sensitivity over a broad bandpass hampers the progress in GRB science. Even in the presence of a redshift determination, with the simple power law description, a key parameter like the overall energy budget of the source cannot be determined, and accurate attempts to model the prompt GRB emission cannot be performed.

The combined use of these satellites with the non-imaging Gamma-ray Burst Monitor (GBM) on board Fermi, or the Wideband All-sky Monitor (WAM) on board Suzaku can improve the quality of GRB prompt data, but the rate of simultaneous detections is small, due to different sensitivities, pointing strategies or earth occultation constraints. Future GRB missions should carry adequate instruments in order to simultaneously obtain prompt localisations and broad band spectroscopy.

Acknowledgements. Based on observations with INTEGRAL, an ESA project with instruments and science data centre funded by ESA member states (especially the PI countries: Denmark, France, Germany, Italy, Switzerland, Spain), Czech Republic and Poland, and with the participation of Russia and the USA. ISGRI has been realized and maintained in flight by CEA-Saclay/Irfu with the support of CNES. This research has been supported by the Italian Space Agency through contract ASI-INAF I/023/05/0. G.V. thanks A. Paizis for helping in the INTEGRAL data reduction and for her effort in solving related problems; M.Turler of the INTEGRAL helpdesk; L.Nava for sharing her knowledge and for interesting discussions. D.G. acknowledges the French Space Agency (CNES) for financial support.

\section{References}

Amati, L. 2006, MNRAS, 372, 233

Amati, L., Frontera, F., Tavani, M., et al. 2002, A\&A, 390, 81

Arnaud, K. A. 1996, in Astronomical Data Analysis Software and Systems V, ed. G. H. Jacoby, \& J. Barnes, ASP Conf. Ser., 101, 17

Band, D., Matteson, J., Ford, L., et al. 1993, ApJ, 413, 281

Beckmann, V., Borkowski, J., Courvoisier, T. J.-L., et al. 2003, A\&A, 411, L327 Blake, C. H., Bloom, J. S., Starr, D. L., et al. 2005, Nature, 435, 181

Bloom, J. S., Kulkarni, S. R., Djorgovski, S. G., et al. 1999, Nature, 401, 453

Bloom, J. S., Frail, D. A., \& Kulkarni, S. R. 2003, ApJ, 594, 674

Blustin, A. J., Campana, S., Nousek, J., White, N., \& Gehrels, N. 2005, GRB Coordinates Network, 4209, 1

Briggs, M. S., Paciesas, W. S., Pendleton, G. N., et al. 1996, ApJ, 459, 40

Cameron, P. B., \& Frail, D. A. 2005, GRB Coordinates Network, 3495, 1

Campana, S., \& Moretti, A. 2004, GRB Coordinates Network, 2656, 1

Capalbi, M., Perri, M., Giommi, P., et al. 2005, GRB Coordinates Network, 3463,1

Castro-Tirado, A. J., Gorosabel, J., Guziy, S., et al. 2003, A\&A, 411, L315

Cenko, S. B., \& Fox, D. B. 2005, GRB Coordinates Network, 3349, 1

Chen, L., Lou, Y.-Q., Wu, M., et al. 2005, ApJ, 619, 983

Cheng, L. X., Ma, Y. Q., Cheng, K. S., Lu, T., \& Zhou, Y. Y. 1995, A\&A, 300, 746

Cobb, B. E., \& Bailyn, C. D. 2008, ApJ, 677, 1157

Cobb, B. E., Bailyn, C. D., van Dokkum, P. G., Buxton, M. M., \& Bloom, J. S. 2004, ApJ, 608, L93

Costa, E., Frontera, F., Heise, J., et al. 1997, Nature, 387, 783

Courvoisier, T. J.-L., Walter, R., Beckmann, V., et al. 2003, A\&A, 411, L53

Covino, S., Antonelli, L. A., Calzoletti, L., et al. 2007, GRB Coordinates Network, 6190, 1

Cummings, J., Barbier, L., Barthelmy, S., et al. 2005, GRB Coordinates Network, 3479, 1

Curran, P. A., van der Horst, A. J., Wijers, R. A. M. J., et al. 2007, MNRAS, 381, L65

D’Avanzo, P., Malesani, D., Campana, S., et al. 2006, ArXiv Astrophysics e-prints

de Luca, A., \& Campana, S. 2005, GRB Coordinates Network, 3109, 1

de Luca, A., Melandri, A., Caraveo, P. A., et al. 2005, A\&A, 440, 85

Della Valle, M., Chincarini, G., Panagia, N., et al. 2006, Nature, 444, 1050

Di Salvo, T., Goldoni, P., Stella, L., et al. 2006, ApJ, 649, L91

Distefano, E., Piranomonte, S., Molinari, E., et al. 2005, GRB Coordinates Network, 4223, 1

Filliatre, P., D’Avanzo, P., Covino, S., et al. 2005, A\&A, 438, 793

Filliatre, P., Covino, S., D’Avanzo, P., et al. 2006, A\&A, 448, 971

Foley, R. J., Chen, H.-W., Bloom, J., \& Prochaska, J. X. 2005, GRB Coordinates Network, 3483, 1

Foley, S., McGlynn, S., Hanlon, L., McBreen, S., \& McBreen, B. 2008, A\&A, 484,143

Fox, D. B., \& React GRB Team. 2005, in BAAS, 37, BAAS, 1418

Frail, D. A. 2003, GRB Coordinates Network, 2473, 1

Frail, D. A. 2005, GRB Coordinates Network, 4350, 1

Frail, D. A., Kulkarni, S. R., Nicastro, L., Feroci, M., \& Taylor, G. B. 1997, Nature, 389, 261

Frail, D. A., Kulkarni, S. R., Sari, R., et al. 2001, ApJ, 562, L55

Fynbo, J. P. U., Greiner, J., Kruehler, T., et al. 2008, GRB Coordinates Network, 8225,1

Gal-Yam, A., Fox, D., Berger, E., \& Wyatt, P. 2004, GRB Coordinates Network, 2555,1

Gehrels, N., Chincarini, G., Giommi, P., et al. 2004, ApJ, 611, 1005

Ghirlanda, G., Bosnjak, Z., Ghisellini, G., Tavecchio, F., \& Firmani, C. 2007, MNRAS, 379, 73

Ghirlanda, G., Nava, L., Ghisellini, G., Firmani, C., \& Cabrera, J. I. 2008, MNRAS, 387, 319

Ghisellini, G., Ghirlanda, G., Mereghetti, S., et al. 2006, MNRAS, 372, 1699

Giommi, P., Capalbi, M., Perri, M., et al. 2005, GRB Coordinates Network, 3054,1

Golenetskii, S., Aptekar, R., Mazets, E., et al. 2005, GRB Coordinates Network, 3474, 1

Golenetskii, S., Aptekar, R., Mazets, E., et al. 2006a, GRB Coordinates Network, 5498,1

Golenetskii, S., Aptekar, R., Mazets, E., et al. 2006b, GRB Coordinates Network, 5841,1

Gorosabel, J., Castro-Tirado, A. J., Tristram, P., et al. 2005, GRB Coordinates Network, 3565, 1 
Götz, D., Mereghetti, S., Hurley, K, et al. 2003, A\&A, 409, 831

Götz, D., Mereghetti, S., \& Mowlavi, N. 2004a, GRB Coordinates Network, 2690, 1

Götz, D., Mereghetti, S., Shaw, S., Beck, M., \& Borkowski, J. 2004b, GRB Coordinates Network, 2866, 1

Grebenev, S. A., \& Chelovekov, I. V. 2007, Astron. Lett., 33, 789

Guidorzi, C., Monfardini, A., Gomboc, A., et al. 2005, ApJ, 630, L121

Guidorzi, C., Romano, P., Vergani, S., \& Moretti, A. 2007a, GRB Coordinates Network, 6192, 1

Guidorzi, C., Vergani, S. D., Sazonov, S., et al. 2007b, A\&A, 474, 793

Halpern, J. 2006, GRB Coordinates Network, 5852, 1

Halpern, J. P., \& Armstrong, E. 2006, GRB Coordinates Network, 5849, 1

Holland, S., Band, D., Blustin, A., et al. 2005, GRB Coordinates Network, 3475, 1

Jelinek, M., de Ugarte Postigo, A., Castro-Tirado, A. J., et al. 2005, GRB Coordinates Network, 4358, 1

Kaneko, Y., Preece, R., Briggs, M., et al. 2006, ApJS, 166, 298

Kennea, J. A., Burrows, D. N., Grupe, D., et al. 2005a, GRB Coordinates Network, 4006, 1

Kennea, J. A., Burrows, D. N., Perri, M., Cummings, J., \& Gehrels, N. 2005b, GRB Coordinates Network, 3434, 1

Kennea, J. A., Burrows, D. N., Zhang, B., \& Gehrels, N. 2005c, GRB Coordinates Network, 3426, 1

Kinugasa, K., \& Torii, K. 2005, GRB Coordinates Network, 4191, 1

Klose, S., Stecklum, B., Fuhrmann, B., Ludwig, F., \& Greiner, J. 2005a, GRB Coordinates Network, 3609, 1

Klose, S., Stecklum, B., Greiner, J., \& Racusin, J. L. 2005b, GRB Coordinates Network, 3611, 1

Klotz, A., Boer, M., \& Atteia, J. L. 2005, GRB Coordinates Network, 3473, 1

Kouveliotou, C., Meegan, C. A., Fishman, G. J., et al. 1993, ApJ, 413, L101

Kuulkers, E., Götz, D., Mereghetti, S., et al. 2004, GRB Coordinates Network, 2699, 1

La Parola, V., Mangano, V., Pagani, C., \& Burrows, D. N. 2005, GRB Coordinates Network, 4338, 1

Lebrun, F., Leray, J. P., Lavocat, P., et al. 2003, A\&A, 411, L141

Lund, N., Budtz-Jørgensen, C., Westergaard, N. J., et al. 2003, A\&A, 411, L231

Malaguti, G., Bazzano, A., Beckmann, V., et al. 2003, A\&A, 411, L307

Malesani, D., Tagliaferri, G., Chincarini, G., et al. 2004a, ApJ, 609, L5

Malesani, D., Tagliaferri, G., Chincarini, G., et al. 2004b, ApJ, 609, L5

Mangano, V., Mineo, T., La Parola, V., et al. 2005, GRB Coordinates Network, 3564, 1

Mas-Hesse, J. M., Giménez, A., Culhane, J. L., et al. 2003, A\&A, 411, L261

McGlynn, S., Clark, D. J., Dean, A. J., et al. 2007, A\&A, 466, 895

Meegan, C. A., Fishman, G. J., Wilson, R. B., et al. 1992, Nature, 355, 143

Mereghetti, S., Götz, D., Beckmann, V., et al. 2003a, A\&A, 411, L311

Mereghetti, S., Götz, D., Borkowski, J., Walter, R., \& Pedersen, H. 2003b, A\&A, 411, L291

Mereghetti, S., Götz, D., Tiengo, A., et al. 2003c, ApJ, 590, L73

Mereghetti, S., Götz, D., Beck, M., et al. 2004a, GRB Coordinates Network, 2670, 1

Mereghetti, S., Götz, D., Beck, M., et al. 2004b, GRB Coordinates Network, 2805, 1

Mereghetti, S., Götz, D., Andersen, M. I., et al. 2005, A\&A, 433, 113

Mineo, T., Campana, S., Chincarini, G., et al. 2005, GRB Coordinates Network, 4210, 1

Mineo, T., Mangano, V., Cusumano, G., \& Sbarufatti, B. 2006, GRB Coordinates Network, 5753, 1

Mitani, T., Barbier, L., Barthelmy, S., et al. 2005, GRB Coordinates Network, 3055,1

Moran, L., Mereghetti, S., Götz, D., et al. 2005, A\&A, 432, 467

Nava, L., Ghisellini, G., Ghirlanda, G., Tavecchio, F., \& Firmani, C. 2006, A\&A, 450,471
Nava, L., Ghirlanda, G., Ghisellini, G., \& Firmani, C. 2008, ArXiv e-prints

Norris, J. P. 2002, ApJ, 579, 386

Norris, J. P., Marani, G. F., \& Bonnell, J. T. 2000, ApJ, 534, 248

Page, K. L., Rol, E., Levan, A. J., et al. 2005, MNRAS, 363, L76

Patel, S., Kouveliotou, C., Mereghetti, S., et al. 2004, GRB Coordinates Network, 2655, 1

Pellizza, L. J., Duc, P.-A., Le Floc'h, E., et al. 2006, A\&A, 459, L5

Perna, R., \& Belczynski, K. 2002, ApJ, 570, 252

Piran, T. 1999, Phys. Rep., 314, 575

Preece, R. D., Briggs, M. S., Mallozzi, R. S., et al. 2000, ApJS, 126, 19

Prochaska, J. X., Bloom, J. S., Chen, H.-W., et al. 2004, ApJ, 611, 200

Prochaska, J. X., Ellison, S., Foley, R. J., Bloom, J. S., \& Chen, H.-W. 2005, GRB Coordinates Network, 3332, 1

Protassov, R., van Dyk, D. A., Connors, A., Kashyap, V. L., \& Siemiginowska, A. 2002, ApJ, 571, 545

Racusin, J., Grupe, D., Hunsberger, S., \& Gehrels, N. 2006, GRB Coordinates Network, 5494, 1

Racusin, J. L., Kennea, J. A., Burrows, D. N., et al. 2005, GRB Coordinates Network, 3610, 1

Rhoads, J. E. 1997, ApJ, 487, L1

Romano, P., Campana, S., Chincarini, G., et al. 2006, A\&A, 456, 917

Ryde, F. 2005, ApJ, 625, L95

Rykoff, E. S., Yost, S. A., \& Swan, H. 2005, GRB Coordinates Network, 3465, 1

Sakamoto, T., Barthelmy, S. D., Barbier, L., et al. 2008, ApJS, 175, 179

Sari, R., \& Piran, T. 1999, ApJ, 520, 641

Sari, R., Piran, T., \& Halpern, J. P. 1999, ApJ, 519, L17

Sazonov, S. Y., Lutovinov, A. A., \& Sunyaev, R. A. 2004, Nature, 430, 646

Sazonov, S. Y., Molkov, S. V., \& Sunyaev, R. A. 2007, Astron. Lett., 33, 656

Schaefer, B. E. 2007, ApJ, 660, 16

Sonoda, E., Maeno, S., Tokunaga, Y., \& Yamauchi, M. 2005, GRB Coordinates Network, 4009, 1

Thompson, C., Mészáros, P., \& Rees, M. J. 2007, ApJ, 666, 1012

Tiengo, A., \& Mereghetti, S. 2006, A\&A, 449, 203

Tiengo, A., Mereghetti, S., \& de Luca, A. 2004, GRB Coordinates Network, 2548,1

Torii, K., \& Mereghetti, S. 2005, GRB Coordinates Network, 4193, 1

Torii, K., Fukazawa, Y., \& Tsunemi, H. 2005, A\&A, 437, L23

Tsutsui, R., Nakamura, T., Yonetoku, D., et al. 2008, MNRAS, 386, L33

Ubertini, P., Lebrun, F., Di Cocco, G., et al. 2003a, A\&A, 411, L131

Ubertini, P., Lebrun, F., Di Cocco, G., et al. 2003b, A\&A, 411, L131

Ulanov, M. V., Golenetskii, S. V., Frederiks, D. D., et al. 2005, Il Nuovo Cimento, 28, 351

van Paradijs, J., Groot, P. J., Galama, T., et al. 1997, Nature, 386, 686

Vanderspek, R., Sakamoto, T., Barraud, C., et al. 2004, ApJ, 617, 1251

Vaughan, S., Willingale, R., O'Brien, P. T., et al. 2004, ApJ, 603, L5

Vergani, S. D., Romano, P., \& Guidorzi, C. 2007, GRB Coordinates Network, 6369, 1

Vestrand, W. T., Wozniak, P. R., Wren, J. A., et al. 2005, Nature, 435, 178

Vietri, M., \& Stella, L. 1998, ApJ, 507, L45

von Kienlin, A., Beckmann, V., Covino, S., et al. 2003, A\&A, 411, L321

Watson, D., Reeves, J. N., Hjorth, J., Jakobsson, P., \& Pedersen, K. 2003, ApJ, 595, L29

Watson, D., Hjorth, J., Levan, A., et al. 2004, ApJ, 605, L101

Wiersema, K., Rol, E., \& Thoene, C. C. 2007, GRB Coordinates Network, 6188, 1

Winkler, C., Courvoisier, T. J.-L., Di Cocco, G., et al. 2003, A\&A, 411, L1

Woosley, S. E. 1993, ApJ, 405, 273

Yonetoku, D., Murakami, T., Nakamura, T., et al. 2004, ApJ, 609, 935

Yost, S. A., Alatalo, K., Rykoff, E. S., et al. 2006, ApJ, 636, 959

Yuan, F., \& Rykoff, E. S. 2006, GRB Coordinates Network, 5759, 1

Zeh, A., Klose, S., \& Hartmann, D. H. 2004, ApJ, 609, 952 

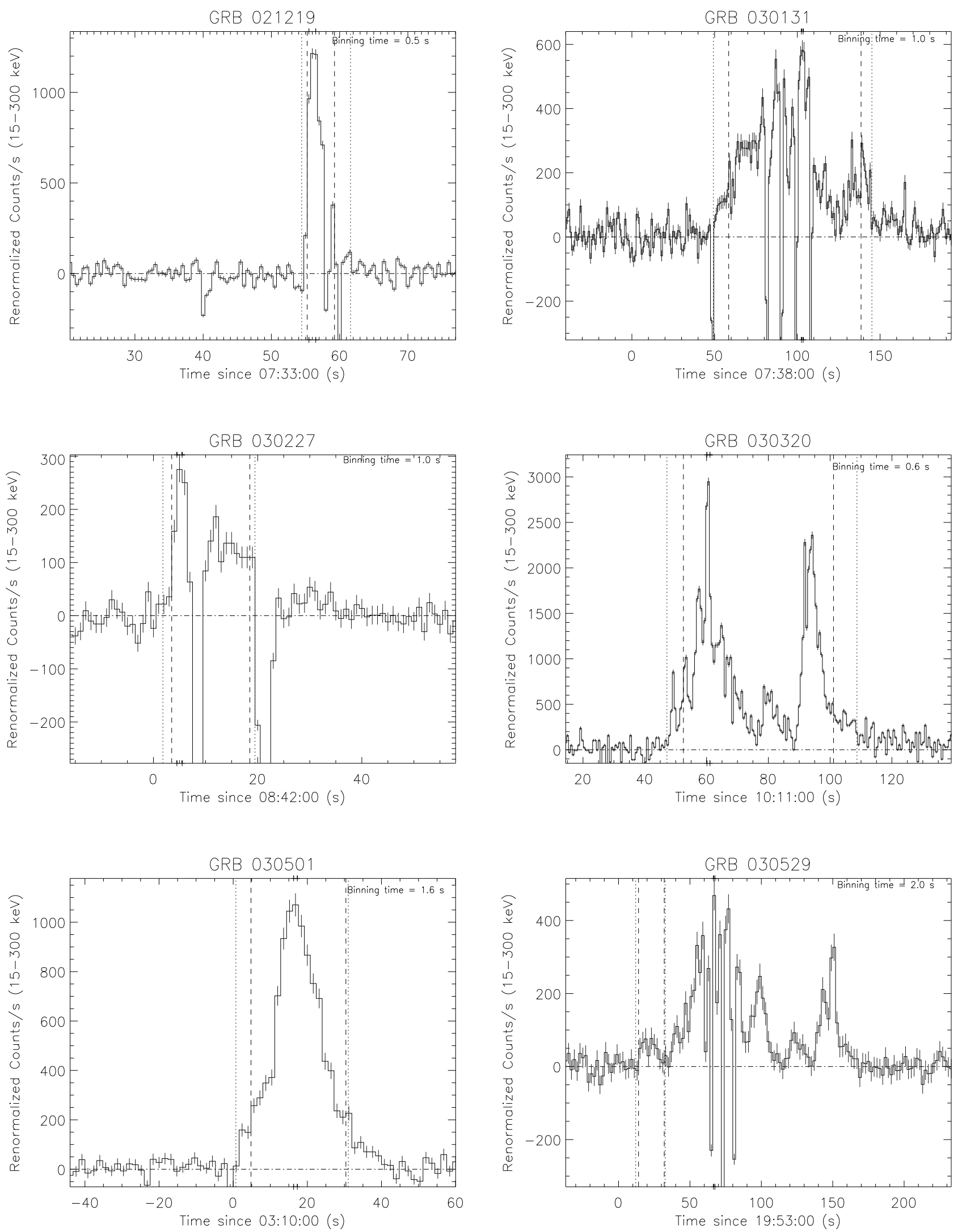

Fig. 12. Light curves of INTEGRAL GRBs. 

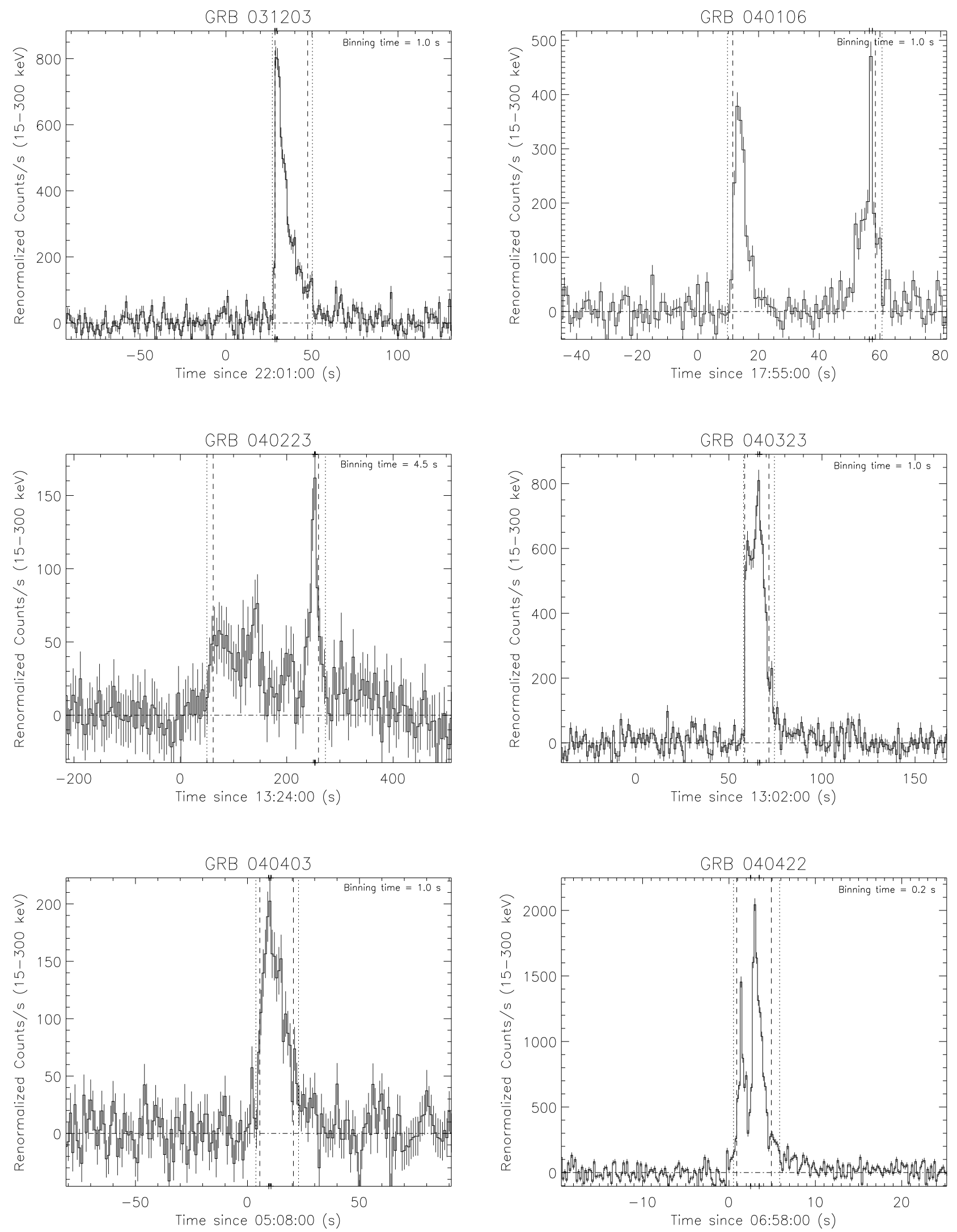

Fig. 12. continued. 

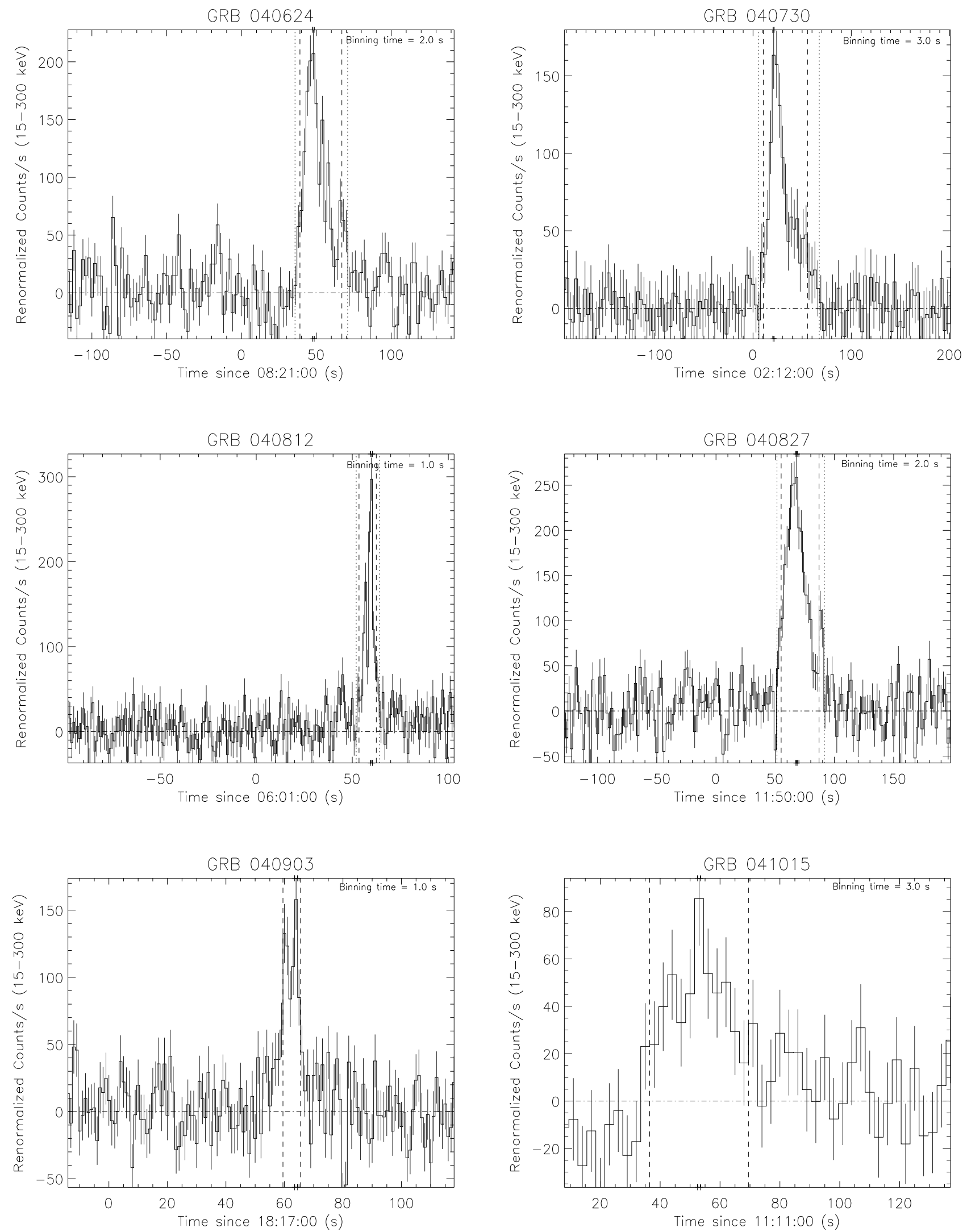

Fig. 12. continued. 

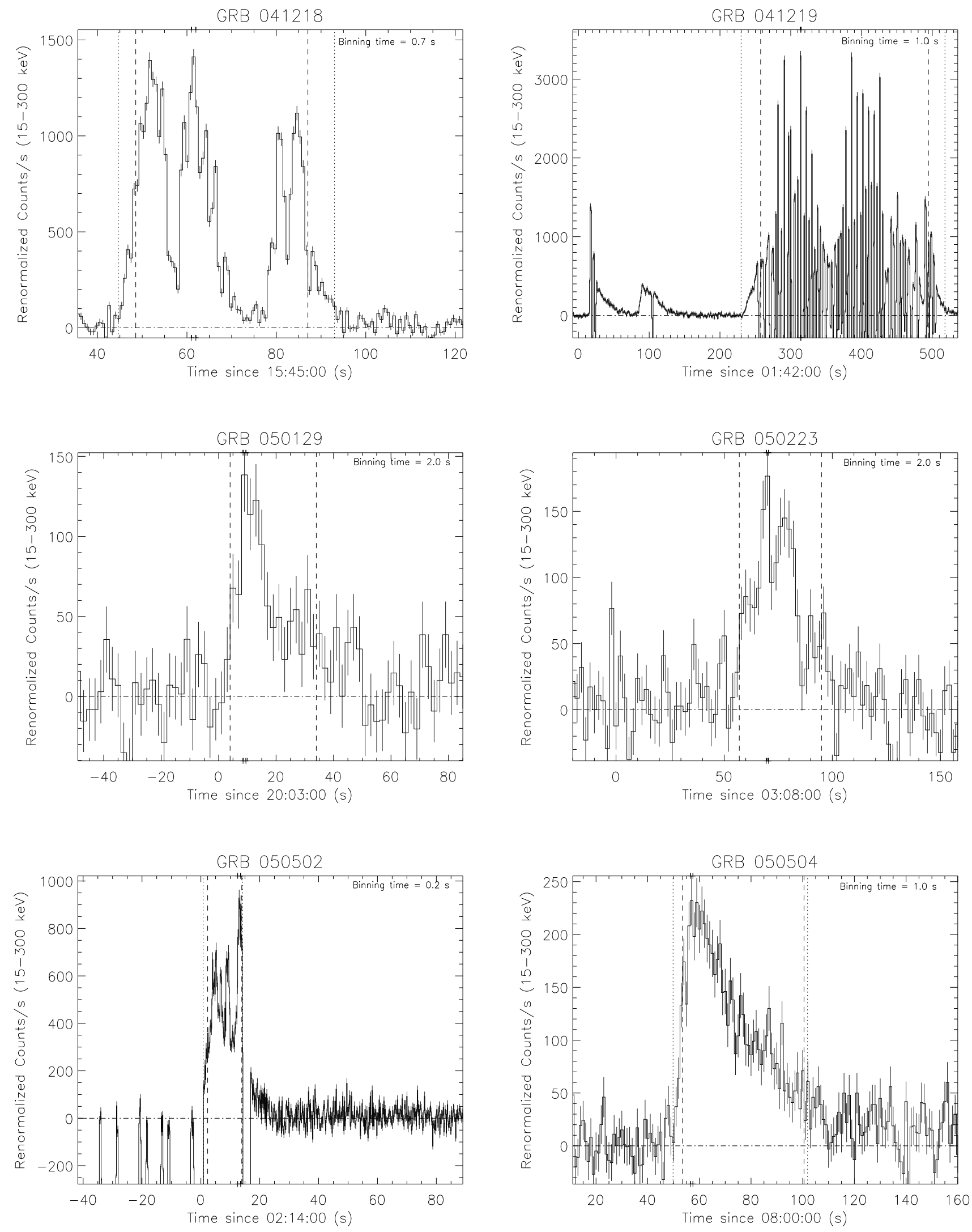

Fig. 12. continued. 

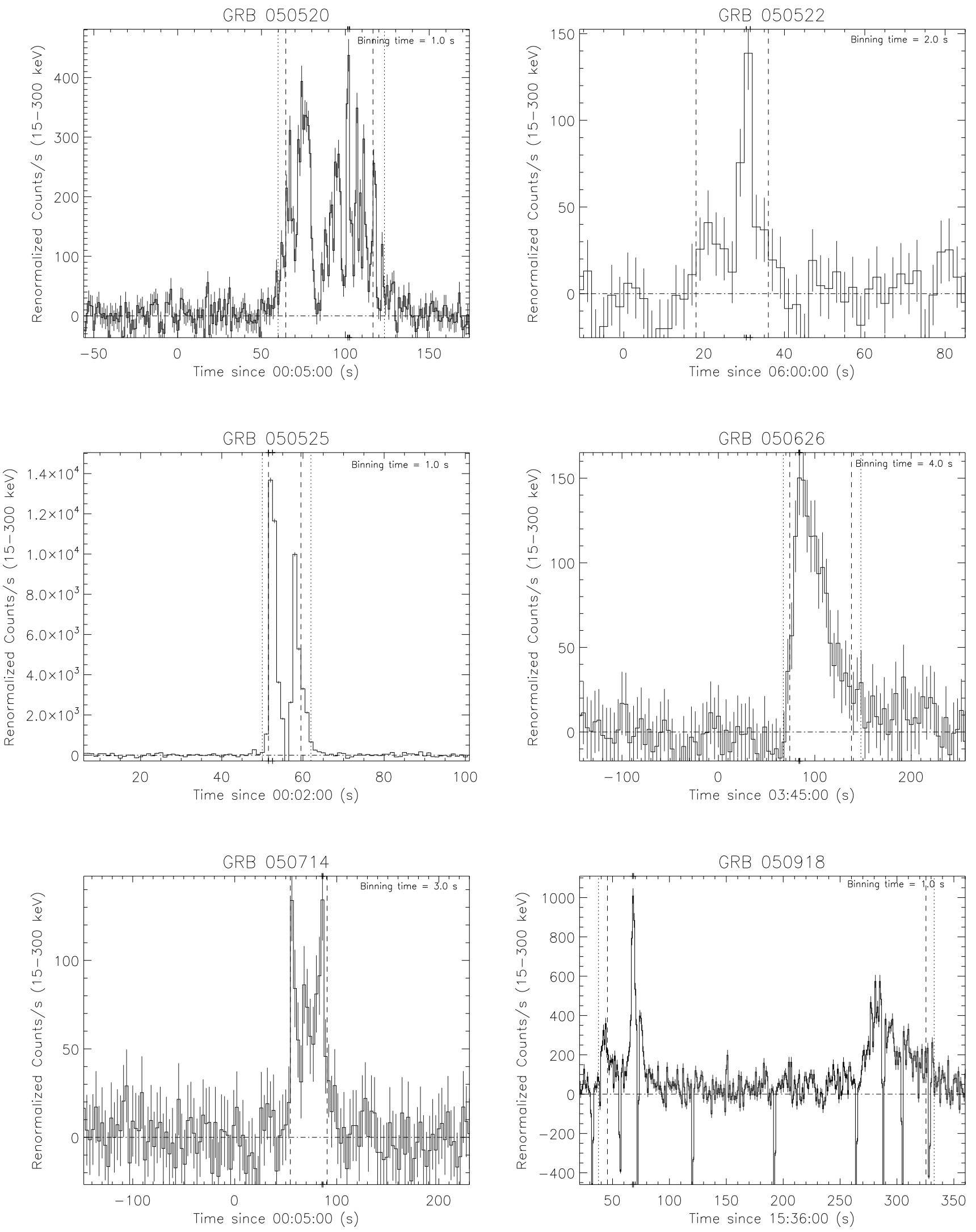

Fig. 12. continued. 

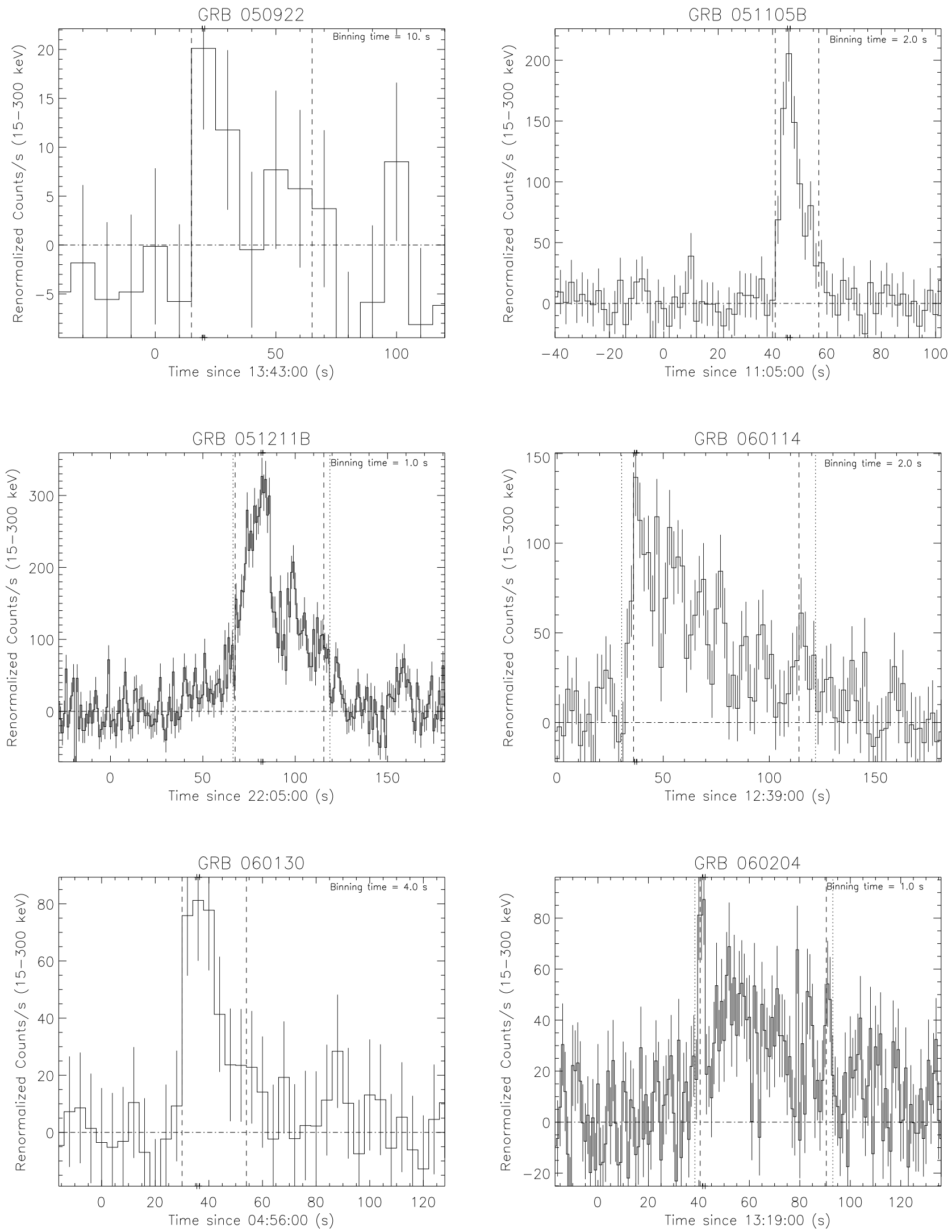

Fig. 12. continued. 

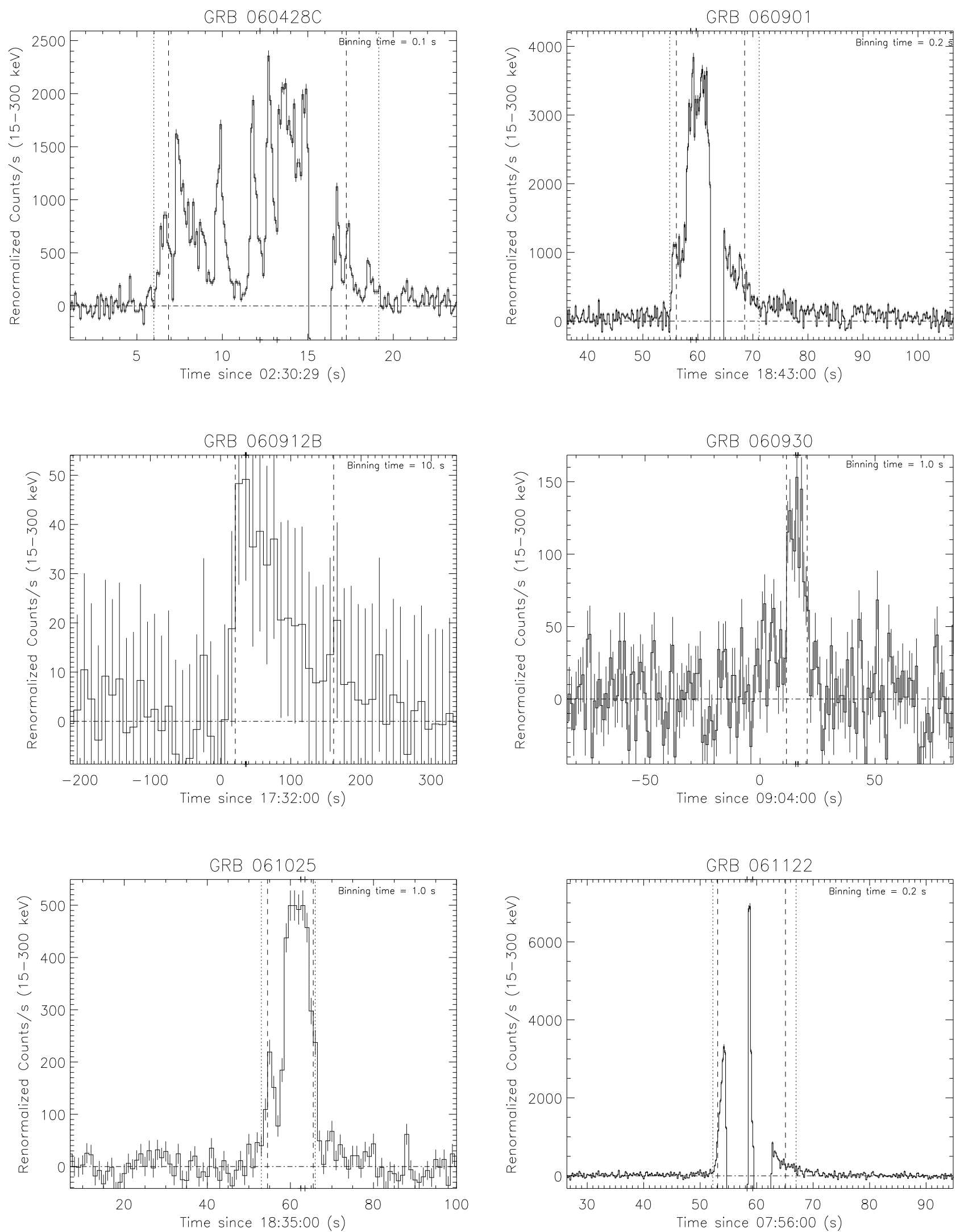

Fig. 12. continued. 

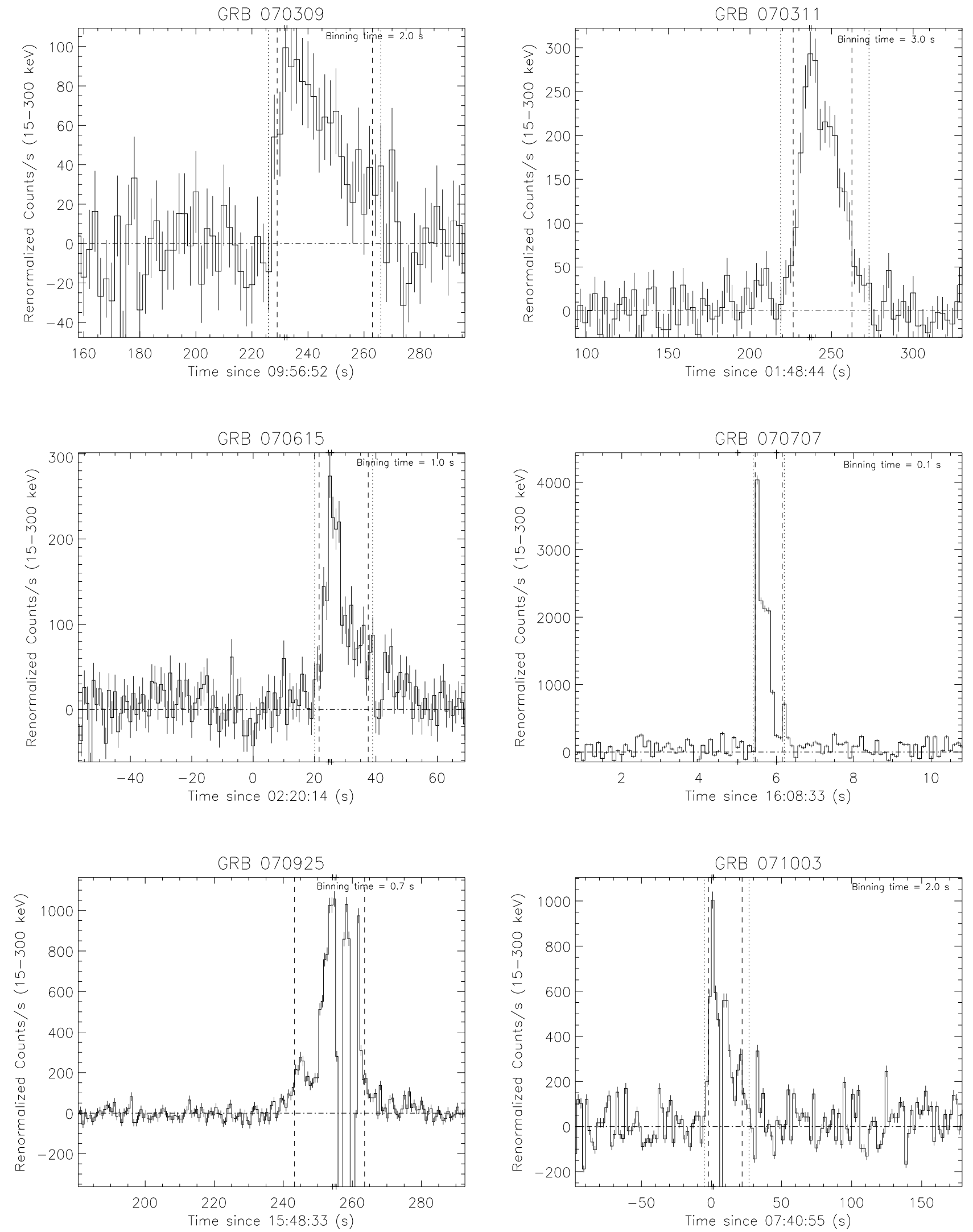

Fig. 12. continued. 


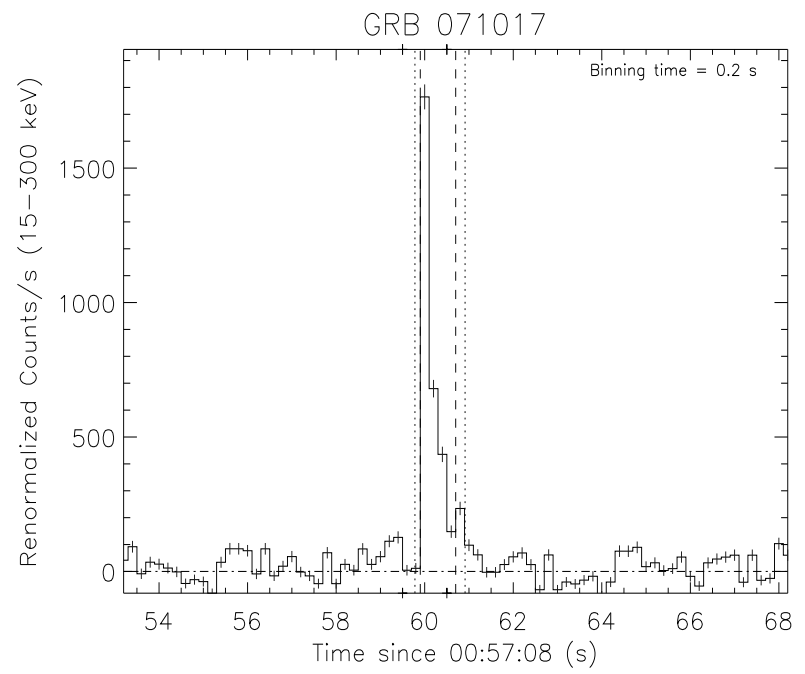

Fig. 12. continued. 